\title{
Dynamic modeling of electrochemical systems using linear graph theory
}

\author{
Thanh-Son Dao*, John McPhee \\ Department of Systems Design Engineering \\ University of Waterloo \\ 200 University Ave West \\ Waterloo, Ontario, Canada N2L $3 G 1$
}

\begin{abstract}
An electrochemical cell is a multidisciplinary system which involves complex chemical, electrical, and thermodynamical processes. The primary objective of this paper is to develop a linear graph-theoretical modeling for the dynamic description of electrochemical systems through the representation of the system topologies. After a brief introduction to the topic and a review of linear graphs, an approach to develop linear graphs for electrochemical systems using a circuitry representation is discussed, followed in turn by the use of the branch and chord transformation techniques to generate final dynamic equations governing the system. As an example, the application of linear graph theory to modeling a nickel metal hydride (NiMH) battery will be presented. Results show that not only the number of equations are reduced significantly, but also the linear graph model simulates faster compared to
\end{abstract}

\footnotetext{
*Corresponding author

Email addresses: tdao@maplesoft.com (Thanh-Son Dao), mcphee@real.uwaterloo.ca (John McPhee)
} 
the original lumped parameter model. The approach presented in this paper can be extended to modeling complex systems such as an electric or hybrid electric vehicle where a battery pack is interconnected with other components in many different domains.

Keywords: linear graph, electrochemical cell, NiMH battery simulation, hybrid electric vehicle. 


\section{List of Figures}

B.1 Electrical network example.

B.2 Linear graph isomorphic to electrical network.

B.3 Electrochemical cell.

B.4 Dependence of current density on surface over-potential on positive electrode of a $\mathrm{NiMH}$ cell at $30^{\circ} \mathrm{C}$.

B.5 Circuitry representation for (a) open-circuit potential equation and (b) Butler-Volmer's equation. . . . . . . . . . .

B.6 Circuitry representation for multiple reactions.

B.7 Equivalent circuit-based representation for electrochemical cell.

B.8 Linear graph representation for (a) chemical domain and (b) thermal domain. . . . . . . . . . . . . . . . . .

B.9 Formulation steps for electrochemical cells.

B.10 Circuitry representation for NiMH cell.

B.11 Linear graph presentation for main and side chemical reactions.

B.12 Linear graph representation for thermal domain. . . . . . . . .

B.13 Battery discharge at constant rates. . . . . . . . . . . .

B.14 Battery charge at constant rates. . . . . . . . . . . .

B.15 Simulated and experimental battery voltages at 1/5 C charge/discharge rate.

B.16 Battery temperature rising from initial temperature $25^{\circ} \mathrm{C}$ at constant discharge rates. . . . . . . . . . . . . .

B.17 Battery temperature rising from initial temperature $25^{\circ} \mathrm{C}$ at constant charge rates. . . . . . . . . . . . . . . . . 


\section{List of Tables}

A.1 Through and across variables. . . . . . . . . . .

B.2 NiMH battery parameters. . . . . . . . . . . . .

B.3 Average simulation time (in seconds) comparison between linear graph model and lumped model. . . . . . . . . . . . . 


\section{Introduction}

Due to the recent interests in battery electric and hybrid electric vehicles, a significant amount of research has been focused on secondary batteries or electrochemical energy storage devices. For this reason, many of these battery works have been developed as a part of simulation models of these vehicles. These works are sometimes based on empirical relationships, at other times on a detailed description of the physical and chemical processes that take place in the cell (Paxton and Newman , 1997; Newman and Tiedemann , 1975; Newman et al. , 2004; Wu et al. , 2001), and even on the development of equivalent circuits (Salameh et al. , 1992; Chen et al. , 2006). Various techniques have been used to develop these models such as lookup tables, lumped parameter models (Wu et al. , 2001), or distributed models using porous electrode theory (Paxton and Newman , 1997; Newman and Tiedemann , 1975; Newman et al. , 2004).

In this paper, we propose a formalism which, we believe, is more appropriate for the phenomenological description of electrochemical systems which usually consists of complex phenomena across multiple domains; namely the chemical domain, electrical domain, thermal domain, and other domains especially when the battery is placed in a larger system such as a hybrid electric vehicle system. Modeling engineers usually cope with the generation and solution of the equations governing the motion of such systems.

Linear graph theory is a branch of mathematics that studies the manipulation of topology (Roe , 1966; Andrews and Kesavan , 1975). Although this theory has been extensively incorporated into formulation of a wide range of physical systems, namely electrical, mechanical, and hydraulic systems, 
the extent to which this theory has been applied to modeling electrochemical and thermal processes remains from nil to minimum. It is the goal of this paper to examine this particular problem in some detail. It will be shown in this paper that the electrochemical processes and thermodynamic behaviors of batteries, in general, can be described as equivalent electrical components interconnected to each other, making it possible to use graph theory to develop the dynamic equations for the whole system.

The paper begins with a brief overview of linear graph theory and associated mathematical theorems, followed by a discussion of the applications of linear graphs to modeling electrochemical cells. An example will also be provided to demonstrate the use of linear graphs to model a NiMH battery including the thermal effects and side reactions. Finally are some concluding remarks.

\section{Linear graph theory}

\subsection{Overview}

A linear graph representation of a physical system is seen as a collection of oriented line segments called edges which intersect only at their node points. Although physical systems in different energy domains use different interpretations of nodes and edges, the linear graph topological interpretations of these systems are the same: nodes are the boundaries of a component, while a set of edges represent the component itself. For example, the linear graph for the electrical network given in Fig. B.1 can be constructed by drawing a node for each point at which two physical elements connect, and by replacing these elements with directed edges on a one-to-one basis. This linear graph 
is shown in Fig. B.2 and is said to be topologically equivalent to the electrical circuit in Fig. B.1. The direction is arbitrarily assigned to each edge and is represented by an arrow which provides a reference direction for the two abstract variables associated with an edge: though variable and across variable.

[Figure 1 about here.]

[Figure 2 about here.]

By definition, a through variable is a variable that can be measured by an instrument in series with the corresponding element associated with the edge, while an across variable is a variable that can be measured by an instrument placed in parallel with the edge. For an electrical network, for example, through and across variables can be the currents and voltages, respectively. A summary of possible through and across variable for some common energy domains are summarized in Table A.1. These two quantities are carried along the entire linear graph so that the balance of energy at any point of the graph can be found. This makes it easier to deal with interfaces between physical systems in different energy domains as well as determining the energy within the system.

The through and across variables of an edge are not independent of each other, but are related by a mathematical expression called terminal equation or constitutive equation which represents the physical nature of the linear graph component. Clearly, the empirical nature of the terminal equation associated with an edge is dependent on the edge's domain. For example, 
the current and voltage associated with an electrical resistor are related by Ohm's law which serves as the terminal equation for the resistor element.

One of the unique features of linear graph theory is its ability to separate the equations governing the physics of a system's individual components from the equations governing their interconnections. That is, the system's topological equations are always linear and may be formulated in a systematic fashion regardless of the linearity/nonlinearity of a system's component equations. The complete topological description of a physical system can be written in a simple mathematical form using an incidence matrix $\boldsymbol{\Gamma}$. This is a $v \times e$ matrix, where $v$ is the number of nodes in the graph, and $e$ is the number of edges. The incidence matrix has elements

$$
\Gamma(i, j)=\left\{\begin{aligned}
-1, & \text { if edge } j \text { is incident upon and towards node } i \\
+1, & \text { if edge } j \text { is incident upon and away from node } i \\
0, & \text { if edge } j \text { is not incident upon node } i
\end{aligned}\right.
$$

Specifically, the directed edge $j$ is positively incident upon node $i$ if it points towards the node, and negatively incident if it is directed away from the node. As an example, the incidence matrix for the linear graph in Fig. B.2 takes the form:

$$
\Gamma={ }_{b}\left[\begin{array}{cccc}
E_{1} & R_{2} & R_{3} & C_{4} \\
1 & 1 & 0 & 0 \\
0 & -1 & 1 & 1 \\
-1 & 0 & -1 & -1
\end{array}\right]
$$

in which the vertices associated with rows and the edges associated with columns have been explicitly labeled. 
An important concept in linear graph theory is the spanning tree, which is a subgraph that includes all the nodes of the original graph without any loops. The remaining edges which are not selected in the tree are grouped in cotrees. The edges of the tree are called branches while the edges of the cotree are referred to as chords. In this paper, a branch is represented by a solid line while a chord is depicted by a dotted line. One possible tree for the graph in Fig. B.2 has been drawn with solid lines and consists of edges $E_{1}$ and $C_{4}$. The remaining edges $R_{2}$ and $R_{3}$ comprise the chords of the cotree. For graphs consisting of multiple parts (as in systems containing multiple energy domains), each part is independently represented by a tree and the collection of all these trees make up the graph's forest, while all of the chords represent its coforest.

Along with the concept of a system tree, two new topological matrices can now be introduced - the fundamental cutset (f-cutset) matrix and the fundamental circuit (f-circuit) matrix. A cutset is defined as a set of edges that, when removed, divide the graph into two separate parts. An f-cutset consists of a single branch and a unique set of chords. On the other hand, a circuit is a set of edges that form a closed loop with the f-circuit being a circuit containing one chord and a unique set of branches. In an electrical system, a cutset is essentially a linear combination of the node-based Kirchoff current law (KCL), which is an expression that the flows passing through a node are conservative. A circuit corresponds to the Kirchoff voltage law (KVL), which sums up the forces operating along the edges enclosing each of the circuits. Mathematical representations of the f-cutset $\left(\mathbf{A}_{f}\right)$ and f-circuit $\left(\mathbf{B}_{f}\right)$ matrices can be written as 


$$
\mathbf{A}_{f} \boldsymbol{\tau}=\left[\begin{array}{ll}
\mathbf{1} & \mathbf{A}_{c}
\end{array}\right]\left[\begin{array}{l}
\boldsymbol{\tau}_{b} \\
\boldsymbol{\tau}_{c}
\end{array}\right]=\mathbf{0}
$$

and

$$
\mathbf{B}_{f} \boldsymbol{\alpha}=\left[\begin{array}{ll}
\mathbf{B}_{b} & \mathbf{1}
\end{array}\right]\left[\begin{array}{l}
\boldsymbol{\alpha}_{b} \\
\boldsymbol{\alpha}_{c}
\end{array}\right]=\mathbf{0}
$$

In these equations, the matrix $\mathbf{1}$ is the identity matrix, $\boldsymbol{\tau}_{b}$ and $\boldsymbol{\tau}_{c}$ represent the branch and chord through variables, and $\boldsymbol{\alpha}_{b}$ and $\boldsymbol{\alpha}_{\mathrm{c}}$ corresponds to the branch and chord across variables. The element $(i, j)$ of the matrix $\mathbf{A}_{c}$ takes on a value of either $+1,-1$, or 0 which indicates whether chord $j$ is a part of and oriented in the same direction as the defining branch $i$, a part of and oriented in the opposite direction of the branch $i$, or not a part of the f-cutset, respectively. In a similar fashion, the value of the element $(i, j)$ of the matrix $\mathbf{B}_{b}$ is either $+1,-1$, or 0 depending whether branch $j$ is a part of and oriented in the same direction along the loop as chord $i$, a part of and oriented in the opposite direction along the loop as chord $i$, or not a part of the f-circuit, respectively.

As a result of its linearity, the cutset equation can be rearranged to express the branch through variables in terms of the chord through variables. This arrangement is called a chord transformation (Roe , 1966; Andrews and Kesavan , 1975; McPhee et al. , 2004; McPhee , 1996, 1998) and can be mathematically expressed as

$$
\boldsymbol{\tau}_{b}=-\mathbf{A}_{c} \boldsymbol{\tau}_{c}
$$

In a similar manner, we may also define the branch transformation (Roe , 1966; Andrews and Kesavan , 1975; McPhee et al. , 2004; McPhee , 1996, 
1998) by rearranging the circuit equation as

$$
\boldsymbol{\alpha}_{c}=-\mathbf{B}_{b} \boldsymbol{\alpha}_{b}
$$

Interestingly, the matrices $\mathbf{A}_{c}$ and $\mathbf{B}_{b}$ are orthogonal as a consequence of the definition of f-cutset and f-circuit matrices (Roe , 1966; Andrews and Kesavan , 1975; Schmitke et al. , 2008)

$$
\mathbf{A}_{c}=-\mathbf{B}_{b}^{T}
$$

\subsection{Formulation of system equations and tree selection}

The f-cutset, f-circuit, and incidence matrices can be used to generate the governing equations for the physical system to which the linear graph is topologically equivalent. In the branch-chord formulation of the system equations, Eqs. (3) and (4) can be used to eliminate the branch through and chord across variables from the set of system equations. A compact set of final equations can be obtained by substituting (3) and (4) into the terminal equations.

As discussed above, the cutset, circuit, and terminal equations provide a necessary and sufficient set of equations for determining the time response of a physical system. Thus, the selection of trees for the graphs does not affect the underlying mathematical model. However, the selection of trees can greatly reduce the number of equations that have to be solved simultaneously, especially if some care is taken in selecting the branches of these trees. Léger and McPhee (2007) made an observation that the number of dynamic equations remaining will depend directly on the number of branch coordinates that have been used. Therefore, the number of equations can be 
reduced further by selecting into the trees those elements for which a minimum number of across variables are unknown. The result will be a smaller number of branch coordinates and therefore, a smaller number of final equations. This observation can also be extended by selecting into the cotrees the edges that can minimize the number of chord through variables so that the number of chord coordinates in the final set of equations is reduced. This approach is useful when we want through variables to appear in the final equations and will be demonstrated in an example given in Section 4 .

Using this simple criterion, it is desirable to include voltage sources in the electrical domain, and position drivers and revolute joints in the mechanical domain, into the trees since their across variables are completely known functions of time. Similarly, current sources in the electrical domain and force actuators in the mechanical domain can be selected into the cotrees since the corresponding through variables appearing in the dynamic equations would be known functions.

\section{Linear graph models for electrochemical cells}

Linear graph theory has been extensively applied to many physical systems in different energy domains, namely mechanical domain, electrical domain, and hydraulic domain (Roe , 1966; Andrews and Kesavan , 1975; McPhee et al. , 2004; McPhee , 1996, 1998; Schmitke et al. , 2008; Léger and McPhee, 2007). Linear graphs have not yet been used to describe electrochemical cells and thermodynamic processes.

In this section, a graph-theoretic representation for electrochemical systems similar to the circuit diagram in electrical network theory will be in- 
troduced. Aside from being intuitively advantageous in presentation, this graphical notation will reveal the role of system topology in dynamic behavior. We will present the procedures for obtaining the dynamic equations governing an electrochemical cell directly from the graph, and consequently one may look upon the network graph as another notation for the differential equations themselves.

\subsection{Batteries and electrochemical processes}

[Figure 3 about here.]

Figure B.3 shows a schematic of a typical electrochemical cell. Every electrochemical system contains two electrodes separated by an electrolyte and connected via an electronic conductor. Ions flow through the electrolyte from one electrode to the other, and the circuit is completed by electrons flowing through the external conductor. At each electrode, an electrochemical reaction is occurring with driving forces for reaction being determined by the thermodynamic properties of the electrodes and electrolyte. In general, a chemical reaction on an electrode can be written as

$$
\sum_{k} s_{k} M_{k}^{z k} \rightleftharpoons \nu \mathrm{e}^{-}
$$

where $M_{k}$ is the symbol for the chemical formula of species $k, s_{k}$ is the stoichiometric coefficient for species $k, \nu$ is the number of electrons, and $z_{k}$ is the original charge of species $k$. For example, consider the reaction

$$
\mathrm{Zn}+2 \mathrm{OH}^{-} \rightleftharpoons \mathrm{ZnO}+2 \mathrm{e}^{-}+\mathrm{H}_{2} \mathrm{O}
$$

In the above chemical equation, $s_{\mathrm{ZnO}}$ is $-1, s_{\mathrm{OH}^{-}}$is $2, z_{\mathrm{ZnO}}$ is $0, z_{\mathrm{OH}^{-}}$is -1 , and $\nu$ is 2 . 
Following historical convention, current is defined as the flow of positive charge. Thus, electrons move in the direction opposite to that of the convention for current flow. Current density is the flux of charge, i.e., the rate of flow of positive charge per unit area perpendicular to the direction of flow. The behavior of electrochemical systems is determined more by the current density than by the total current, which is the product of the current density and the surface area of the porous electrode. In this paper, symbol $j$ refers to current density.

Owing to the historical development of the field of electrochemistry, we use over-potential to refer to the magnitude of the potential drop caused by the resistance to the passage of current and open-circuit potential for the potential between two battery electrodes at which no current flows. This open-circuit potential $\phi(t)$ is derived from the Gibbs free energy and then is reduced to the Nernst equation as

$$
\phi(t)=U+\left(T(t)-T_{0}\right) \frac{\partial U}{\partial T}-\frac{R T(t)}{\nu F} \ln \left(\prod_{k} c_{k}^{s_{k}}(t)\right)
$$

where $U$ is the open-circuit potential at standard conditions for the electrode, $T_{0}$ is the reference temperature, $\frac{\partial U}{\partial T}$ is the reversible heat constant for the reaction, and $c_{k}(t)$ is the concentration or molality of the reactant $k$. The molality $c_{k}(t)$ can be related to the electric charge $q_{k}(t)$ using Faraday's law:

$$
c_{k}(t)=\frac{q_{k}(t)}{2 F}
$$

while the relationship between charge and current can be represented by

$$
i_{k}(t)=\frac{d q_{k}(t)}{d t}
$$


The other parameters $F, R$, and $T$ are the Faraday constant, the gas constant, and the battery temperature, respectively.

The resistive force for the chemical reactions at the two electrodes is termed the surface over-potential and is given the symbol $\eta$. The current density $j(t)$, which is directly related to the rate of chemical reaction, can be expressed as the function of the surface over-potential by the Butler-Volmer equation in the form

$$
\begin{aligned}
j(t) & =i_{0}\left[\exp \left(\frac{\alpha F}{R T(t)} \eta(t)\right)-\exp \left(-\frac{\alpha F}{R T(t)} \eta(t)\right)\right] \times \exp \left[\frac{E_{a}}{R}\left(\frac{1}{T(t)}-\frac{1}{T_{0}}\right)\right] \\
& =2 i_{0} \sinh \left(\frac{\alpha F}{R T(t)} \eta(t)\right) \times \exp \left[\frac{E_{a}}{R}\left(\frac{1}{T(t)}-\frac{1}{T_{0}}\right)\right]
\end{aligned}
$$

The Arrhenius equation $\exp \left[\frac{E_{a}}{R}\left(\frac{1}{T(t)}-\frac{1}{T_{0}}\right)\right]$ represents the dependency of the reaction rate on the battery temperature $T(t)$ and the activation energy $E_{a}$. A positive $\eta(t)$ produces a positive (anodic) current. The derivation and application of the Butler-Volmer equation, and its limitations, is discussed in Chapter 8 in the work of Newman et al. (2004). The dimensionless parameter $\alpha$, called charge transfer coefficient is an additional kinetic parameter that relates how an applied potential favors one direction of reaction over the other. It usually has a value between 0.2 and 2.0. The parameter $i_{0}$ is called the exchange current density and is analogous to the rate constant used in chemical kinetics. A reaction with a large value of $i_{0}$ is often called fast or reversible. As an example, the relationship between the current density $j(t)$ and the surface over-potential $\eta(t)$ for the main chemical reaction on the positive electrode in a NiMH cell is graphed in Fig. B.4.

[Figure 4 about here.] 
Besides the main chemical reaction that generates most of the battery's current, there are usually several side reactions happening in the cell container. The empirical nature of these side chemical reactions depends on the type of batteries. For example, the side reactions are the hydrogen evolution and absorbtion reactions in a lead-acid battery and the oxygen evolution and absorbtion reactions in a NiMH cell. These side reactions may have a significant impact on the battery performance and, therefore, modeling these effects is desirable. Mathematically, modeling side reactions is similar to that of the main reaction since they are all chemical reactions.

\subsection{Electrical circuit-based representation for chemical reactions}

To understand the composition of the model, an electrical circuit with equivalent components will be developed. Through the relations between electrical components, this equivalent circuit will clearly show the relationship between the electrochemical equations to facilitate the application of linear graph theory to the system.

The first equation we will examine is the open-circuit potential equation (i.e., the Nernst equation), Eq. (8), which relates the electrode open-circuit potential $\phi(t)$ to the molality of the substances. Since molality and electric charge are directly related by Faraday's laws in (9), Eq. (8) can be thought of as a nonlinear electrical capacitor (Fig. B.5a) which stores free energy, integrates the current density $j(t)$ in order to obtain the electric charge, and thereby obtains the open-circuit potential. Starting off from the initial battery state of charge which is defined by the molality of materials on the battery electrodes, the charge gradually increases/decreases when the cell is charged/discharged and so does the stored electrochemical energy. The 
chemical kinetics represented by the Butler-Volmer equation in (11) can be modeled by a nonlinear electrical resistor in which the current density $j(t)$ and the surface over-potential $\eta(t)$ are coupled by a nonlinear expression. This component is illustrated in Fig. B.5b.

[Figure 5 about here.]

Since the current density in Eq. (11) is the current density that moves the ions (i.e., the molalities in Eq. (8)) from one electrode to another, these two components must be connected in series. The total electrical potential across both of these components is the sum of the individual potentials across each of the two components and is called the positive or negative electrode potential. We can state that: each chemical reaction is represented by one pair of these capacitor and resistor. If we only consider the main chemical reactions, there will be two pairs of capacitor and resistor, each representing a chemical reaction on one electrode.

Now let us consider an electrode at which there are $n$ chemical reactions (i.e., both main and side reactions). Since these reactions are independent of each other, the total electrode current density $j_{\text {total }}(t)$ is obtained by adding up the current densities of the individual reactions. That is

$$
j_{\text {total }}(t)=j_{1}(t)+j_{2}(t)+\ldots+j_{n}(t)=\frac{i_{\text {cell }}}{A_{\text {surf }}} .
$$

where $A_{\text {surf }}$ is the surface area of the porous electrode and $i_{\text {cell }}$ is the current produced by the battery. The voltage across each capacitor-resistor pair is also the same

$$
\phi_{C_{1}}(t)+\eta_{R_{1}}(t)=\phi_{C_{2}}(t)+\eta_{R_{2}}(t)=\ldots=\phi_{C_{n}}(t)+\eta_{R_{n}}(t)
$$


It can be inferred from Eqs. (12) and (13) that the electrode can be represented by multiple capacitor-resistor pairs hooked up in parallel as shown in Fig. B.6.

[Figure 6 about here.]

We can close the circuit by connecting the positive and negative terminals to a current source or an external load as shown in Fig. B.7. The external load could be a complete electric vehicle. In this figure, $R_{\text {int }}$ is the internal resistance of the battery. For some batteries, this resistance is very small and its effects can be ignored.

[Figure 7 about here.]

\subsection{Thermal effects}

So far, we have presented the electrical circuit representation for the chemical reactions in an electrochemical cell. The model that we consider assumes that the battery temperature is constant or, in other words, the battery model we have investigated so far is an isothermal model. This assumption is generally acceptable for small cells where the applied current is not high. However, when the current intensity is high, as in the case of traction batteries for electric or hybrid electric vehicles, the effects of battery temperature can become significant.

Application of energy balance (Wang , 1999; Gomadam , 2003) to the whole cell yields

$c_{p} m_{\text {cell }} \frac{d T(t)}{d t}=-h A_{\text {cell }}\left(T(t)-T_{a}\right)+i_{\text {cell }}(t) v_{\text {cell }}(t)-\sum_{k=1}^{n} j_{k}(t)\left(\phi_{k}(t)-T(t) \frac{\partial U_{k}}{\partial T}\right)$ 
In this equation, $c_{p}$ is the heat capacity of the cell, $m_{\text {cell }}$ is the mass of the cell, $h$ is the external heat transfer coefficient, $A_{\text {cell }}$ is the cell container external surface area, $T_{a}$ is the ambient temperature, and $n$ is the number of chemical reactions. The right-hand side of the equation consists of three terms: the first term corresponds to the heat exchange with the outside environment through the cell container walls according to Newton's law of cooling, the second term refers to the irreversible heat arisen from ohmic heating for the whole cell, and the last term is the reversible entropic heat released or absorbed by the chemical reactions. Equation (14) shows that the heat generation rate is equal to the sum of heat transferred out of the system and the heat stored in the system.

According to classical thermodynamics, Eq. (14) can be written as

$$
\frac{d Q(t)}{d t}=\frac{d Q_{\mathrm{ext}}(t)}{d t}+\frac{d Q_{\mathrm{irr}}(t)}{d t}+\frac{d Q_{\mathrm{rev}}(t)}{d t}
$$

in which $d Q(t)=c_{p} m_{\text {cell }} d T(t)$ according to the definition of heat capacity and $\frac{d Q_{\mathrm{ext}}(t)}{d t}=-h A_{\mathrm{cell}}\left(T(t)-T_{a}\right)$ according to Newton's law of cooling. The last two terms, $\frac{d Q_{\mathrm{irr}}(t)}{d t}=i_{\text {cell }}(t) v_{\text {cell }}(t)$ and $\frac{d Q_{\mathrm{rev}}(t)}{d t}=-\sum_{k=1}^{n} j_{k}(t)\left(\phi_{k}(t)-T(t) \frac{\partial U_{k}}{\partial T}\right)$, are the rates of heat dissipated/absorbed due to the internal resistance and chemical reactions. In electrochemistry, $Q_{\text {rev }}(t)$ is also called the Gibbs freeenergy change.

In order to develop a linear graph for the thermal domain, we need to transform the thermal balance equation into the temperature-entropy form so that the through $\dot{S}(t)$ and across $T(t)$ variables appear explicitly in the equation. Dividing both sides of Eq. (15) by $T(t)$ results in

$$
\dot{S}(t)=\dot{S}_{\text {ext }}(t)+\dot{S}_{\text {irr }}(t)+\dot{S}_{\text {rev }}(t)
$$


where $\dot{S}_{\text {ext }}(t), \dot{S}_{\text {irr }}(t)$, and $\dot{S}_{\text {rev }}(t)$ are the time derivatives of the entropy for the external temperature exchange term, the irreversible term, and the reversible term, respectively. In Eq. (16), $\dot{S}_{\text {rev }}(t)$ is the sum of the individual entropies for the chemical reactions and can be expressed as $\dot{S}_{\mathrm{rev}}(t)=\sum_{k=1}^{n} \dot{S}_{\mathrm{rev}_{k}}(t)$.

\subsection{Linear graph for battery model}

Following the circuitry representation for the chemical domain as discussed in Section 3.2 and the temperature-entropy representation given in Section 3.3, we can develop the linear graphs for both domains. Examples for such graphs are shown in Fig. B.8. In this figure, $C$ 's and $R$ 's are the nonlinear capacitors and resistors whose equations are given in Eq. (8) and Eq. (11). For convenience, the current $i(t)$ and voltage $v(t)$ will be used as through and across variables for the chemical domain. The current flowing through each component can be related to the current density by $i(t)=A_{\text {surf }} j(t)$. The voltage across each resistor is the surface-over potential $\eta(t)$ while the voltage across the each capacitor component is the open-circuit voltage $\phi(t)$. The tree branches and chords have been arbitrarily chosen as shown in Fig. B.8a, which bears a striking resemblance to the physical system in Fig. B.7. We use a solid line to represent a tree branch and a dotted line for a chord. If the model of the external circuit is known, we can also construct the linear graph for the entire system. For battery charge and discharge operations, the external circuit is simply a current source which delivers electric current to the battery. The two graphs in Fig. B. 8 are coupled by the temperature variable $T(t)$ which appears in Eqs. (8) and (11).

[Figure 8 about here.] 
For the thermal domain, the graph is simply a set of edges connected in parallel. The through and across variables for the thermal domain are the time-derivative of entropy $\dot{S}(t)$ and the battery temperature $T(t)$, respectively. It can be realized that the product of $\dot{S}(t)$ and $T(t)$ is power, same as the product of voltage and current. This indicates that the energy flowing through the system components is conserved.

The system dynamic equations can be developed following the procedures discussed in Section 2.2. We can write the through variables vector for the chemical domain as

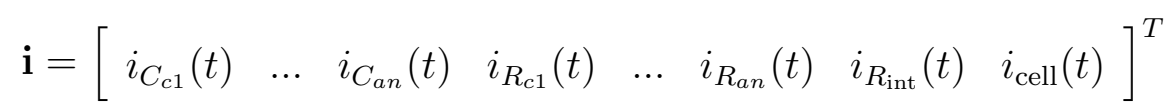

The molalities $c_{k}$ 's in Eq. (8) can be replaced by charge variables $q_{k}$ 's using Faraday's law in (9). These charges are also through variables

$$
\mathbf{q}=\left[\begin{array}{llllllll}
q_{C_{c 1}}(t) & \ldots & q_{C_{a n}}(t) & q_{R_{c 1}}(t) & \ldots & q_{R_{a n}}(t) & q_{R_{\mathrm{int}}}(t) & q_{\text {cell }}(t)
\end{array}\right]^{T}
$$

Similarly, the across variable vector can be defined as follows

$$
\mathbf{v}=\left[\begin{array}{llllllll}
v_{C_{c 1}}(t) & \ldots & v_{C_{a n}}(t) & v_{R_{c 1}}(t) & \ldots & v_{R_{a n}}(t) & v_{R_{\mathrm{int}}}(t) & v_{\text {cell }}(t)
\end{array}\right]^{T}
$$

We can also define the through and across variable vectors for the thermal domain

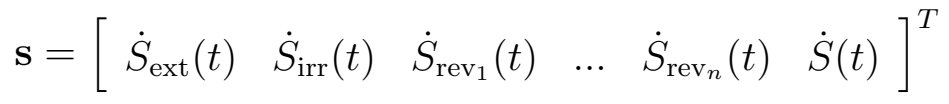

and

$$
\mathbf{t}=\left[\begin{array}{llllll}
T_{\mathrm{ext}}(t) & T_{\mathrm{irr}}(t) & T_{\mathrm{rev}_{1}}(t) & \ldots & T_{\operatorname{rev}_{n}}(t) & T(t)
\end{array}\right]^{T}
$$


The branch and chord transformations can be applied directly to the current and voltage variables as shown in Eqs. (4) and (3). However, for the charge variables, initial values, which appear as we integrate the current variables, must be included in the chord transformation equation. This gives

$$
\left[\mathbf{q}_{b}(t)+\mathbf{q}_{b}(0)\right]=-\mathbf{A}_{c q}\left[\mathbf{q}_{c}(t)+\mathbf{q}_{c}(0)\right]
$$

The formulation procedures for an electrochemical system can be summarized as in Fig. B.9. The figure depicts the steps in the formulation as well as their resulting output variables. After the final step we obtain $2(n-1)+1$ ODEs representing the charge-current relation equations, $2(n-1)+1$ algebraic equations for the chemical domain, and 1 ODE for the thermal domain. It can be observed that $2(n-1)+1$ is also the number of chords in the linear graph. The number of equations can be reduced further if some of the through variables are known functions of time. As an example, if the applied current on the battery terminals is known then $i_{\text {cell }}$ can be considered as a current driver and two equations (i.e., one charge-current relation and one algebraic equation) can be eliminated from the final equations since the value of $i_{\text {cell }}$ can now be substituted directly into all the equations.

[Figure 9 about here.]

\section{Application to NiMH cell model}

Due to its dominance in almost all hybrid vehicles the NiMH battery model has been chosen to demonstrate the technique in this paper. The $\mathrm{NiMH}$ is one of the latest battery technologies and has many advantages 
over the other more commonly used rechargeable batteries such as the leadacid battery or the nickel-cadmium battery. Some of these advantages include higher energy density, more environmental friendly, and less prone to memory (i.e., periodic exercise cycles need to be done less often). The NiMH battery model presented is a modified version of the lumped battery model proposed by $\mathrm{Wu}$ et al. (2001). In this section, the NiMH chemistry together with side reactions and thermal effects will be presented, followed by a linear-graphbased formulation for both chemical and thermal domains.

\subsection{NiMH battery chemistry}

The chemical reactions on the two electrodes of the battery can be written as follows:

Main reaction on positive electrode:

$$
\mathrm{NiOOH}+\mathrm{H}_{2} \mathrm{O}+\mathrm{e}^{-} \underset{\text { charge }}{\stackrel{\text { discharge }}{\rightleftharpoons}} \mathrm{Ni}(\mathrm{OH})_{2}+\mathrm{OH}^{-}
$$

Side reaction on positive electrode:

$$
2 \mathrm{OH}^{-} \rightarrow \frac{1}{2} \mathrm{O}_{2}+\mathrm{H}_{2} \mathrm{O}+2 \mathrm{e}^{-}
$$

Main reaction on negative electrode:

$$
\mathrm{MH}+\mathrm{OH}^{-} \underset{\text { charge }}{\stackrel{\text { discharge }}{\rightleftharpoons}} \mathrm{M}+\mathrm{H}_{2} \mathrm{O}+\mathrm{e}^{-}
$$

Side reaction on negative electrode:

$$
\frac{1}{2} \mathrm{O}_{2}+\mathrm{H}_{2} \mathrm{O}+2 \mathrm{e}^{-} \rightarrow 2 \mathrm{OH}^{-}
$$

where the metal $\mathrm{M}$ in the negative electrode is an inter-metallic compound, usually a rare earth compound. During charging, oxygen is generated at 
the nickel electrode and the gas is formed when the solubility limit in the electrolyte is reached. The oxygen is then transported to the metal hydride electrode where it is reduced by the recombined reaction (26). During discharge, the oxygen generation reaction may occurs at low rates, at which the potential of the nickel electrode is higher than the equilibrium potential of the oxygen generation ( $\mathrm{Gu}$ et al. , 1999; Paul et al. , 2008).

The electromotive force in the battery as defined by the open-circuit potentials (i.e., Nernst's equations) for the main reactions $(23,25)$ on the positive and negative electrodes is

$$
\begin{aligned}
& \phi_{1}(t)=U_{1}+\left(T(t)-T_{0}\right) \frac{\partial U_{1}}{\partial T}+\frac{R T(t)}{F} \ln \left(\frac{c_{\mathrm{H}^{+}, \text {max }}-c_{H^{+}}(t)}{c_{e} c_{\mathrm{H}^{+}}(t)}\right) \\
& \phi_{3}(t)= U_{3}+\left(T(t)-T_{0}\right) \frac{\partial U_{3}}{\partial T}+\frac{R T(t)}{F} \ln \left(c_{e}^{2}\right)+9.712 \times 10^{-4} \\
&+0.2372 \exp \left(-\frac{28.057 c_{\mathrm{MH}}(t)}{c_{\mathrm{MH}, \max }}\right)-\frac{2.7302 \times 10^{-4}}{\left(\frac{c_{\mathrm{MH}}(t)}{c_{\mathrm{MH}, \max }}\right)^{2}+0.010768}
\end{aligned}
$$

and the equilibrium potential for the oxygen reactions $(24,26)$ is given by

$$
\phi_{2}(t)=U_{2}+\left(T(t)-T_{0}\right) \frac{\partial U_{2}}{\partial T}+\frac{R T(t)}{2 F} \ln \left(\frac{p_{\mathrm{O}_{2}}^{0.5}(t)}{c_{e}^{2}}\right)
$$

In these equations, $c_{\mathrm{H}^{+}}(t)$ is the concentration of $\mathrm{Ni}(\mathrm{OH})_{2}, c_{\mathrm{MH}}(t)$ is the concentration of the metal hydride $(\mathrm{MH}), p_{\mathrm{O}_{2}}$ is the partial pressure of oxygen gas, and $T(t)$ is the battery temperature. Other parameters and constants are listed in Table B.2. Equation (28) was curve-fitted from the experimental data of a nickel/KOH $/ \mathrm{LaNi}_{5}$ battery using a nickel oxide positive electrode provided by Paxton and Newman (1997). There is only one open-circuit potential equation (29) for the two side reactions $(24,26)$ since side reactions 
are coupled together by the oxygen transport from the positive electrode to the negative electrode.

The rate of chemical reactions on each electrode is defined by the ButlerVolmer equation which relates the current density $j_{k}(t)$ to the surface overpotential $\eta_{k}(t)$ by

$$
j_{k}(t)=i_{0, k}\left[\exp \left(\frac{\alpha_{k} F}{R T(t)} \eta_{k}(t)\right)-\exp \left(-\frac{\alpha_{k} F}{R T(t)} \eta_{k}(t)\right)\right]
$$

where $k=1 . .3$ for the first three chemical equations (23-25). The exchange current density $i_{0, k}$ for each chemical equation is given by

$$
\begin{gathered}
i_{0,1}=i_{0,1, \text { ref }}\left(\frac{c_{\mathrm{H}^{+}}(t)}{c_{\mathrm{H}^{+}, \text {ref }}}\right)^{0.5}\left(\frac{c_{e}}{c_{e, \text { ref }}}\right)^{0.5}\left(\frac{c_{\mathrm{H}^{+}, \text {max }}-c_{\mathrm{H}^{+}}(t)}{c_{\mathrm{H}^{+}, \text {max }}-c_{\mathrm{H}^{+}, \text {ref }}}\right)^{0.5} \exp \left[\frac{E_{a, 1}}{R}\left(\frac{1}{T(t)}-\frac{1}{T_{0}}\right)\right] \\
i_{0,2}=i_{0,2, \text { ref }}\left(\frac{c_{e}}{c_{e, \text { ref }}}\right)^{0.5}\left(\frac{p_{\mathrm{O}_{2}}(t)}{p_{\mathrm{O}_{2}, \text { ref }}}\right)^{0.5} \exp \left[\frac{E_{a, 2}}{R}\left(\frac{1}{T(t)}-\frac{1}{T_{0}}\right)\right] \\
i_{0,3}=i_{0,3, \text { ref }}\left(\frac{c_{\mathrm{MH}}(t)}{c_{\mathrm{MH}, \text { ref }}}\right)^{0.5}\left(\frac{c_{e}}{c_{e, \text { ref }}}\right)^{0.5}\left(\frac{c_{\mathrm{MH}, \text { max }}-c_{\mathrm{MH}}(t)}{c_{\mathrm{MH}, \text { max }}-c_{\mathrm{MH}, \text { ref }}}\right)^{0.5} \exp \left[\frac{E_{a, 3}}{R}\left(\frac{1}{T(t)}-\frac{1}{T_{0}}\right)\right]
\end{gathered}
$$

where $i_{0, k, r e f}$ is the exchange current density at a reference reactant concentration.

For the oxygen reduction reaction on the negative electrode, a limiting current equation is used for the rate of reaction

$$
j_{4}(t)=-\frac{p_{\mathrm{O}_{2}}(t)}{p_{\mathrm{O}_{2}, \text { ref }}} i_{0,4}
$$

where

$$
i_{0,4}=i_{0,4, \text { ref }} \exp \left[\frac{E_{a, 4}}{R}\left(\frac{1}{T(t)}-\frac{1}{T_{0}}\right)\right]
$$


The battery current $i_{\text {cell }}(t)$ can be calculated from the charge balance equations on the electrodes given by

$$
\begin{gathered}
i_{\text {cell }}(t)=A_{\text {pos }} a_{\text {pos }} l_{\text {pos }}\left(j_{1}(t)+j_{2}(t)\right) \\
i_{\text {cell }}(t)=-A_{\text {neg }} a_{\text {neg }} l_{\text {neg }}\left(j_{3}(t)+j_{4}(t)\right)
\end{gathered}
$$

The mass balance of the nickel active material is given by

$$
\begin{gathered}
j_{1}(t)=F \frac{d c_{\mathrm{H}^{+}}(t)}{d t} \frac{L_{\mathrm{Ni}(\mathrm{OH})_{2}}}{\rho_{\mathrm{Ni}(\mathrm{OH})_{2}} l_{\mathrm{pos}} a_{\mathrm{pos}}} \\
j_{3}(t)=F \frac{d c_{\mathrm{MH}}(t)}{d t} \frac{L_{\mathrm{MH}}}{\rho_{\mathrm{MH}} l_{\mathrm{neg}} a_{\mathrm{neg}}} \\
A_{\mathrm{pos}} a_{\mathrm{pos}} l_{\mathrm{pos}} j_{2}(t)+A_{\mathrm{neg}} a_{\mathrm{neg}} l_{\mathrm{neg}} j_{4}(t)=F \frac{d p_{\mathrm{O}_{2}}(t)}{d t} \frac{V_{\mathrm{gas}}}{R T(t)}
\end{gathered}
$$

The battery temperature can be obtained from the energy balance of the whole cell described by Eq. (14).

\subsection{Linear graph representation for chemical domain}

The application of the linear graph concept to the chemical reactions of the NiMH battery is a straightforward operation. The main and side chemical reactions for the NiMH battery model shown in Section 4.1 can be represented by an equivalent electrical circuit as shown in Fig. B.10. The open-circuit voltage equations (27), (29), and (28) can be represented by nonlinear electrical capacitors $C_{1}, C_{2}$, and $C_{3}$. The relationship between the electrical potentials $\phi_{k}$ and concentrations in these equations is similar to the capacitive relationship between voltage and charge in an electrical capacitor.

The nonlinear resistors $R_{1}, R_{2}$, and $R_{3}$ are used to model the resistive relationship between the current density and over voltage in Eq. (30). The 
current density for the oxygen reduction reaction in Eq. (34) and the applied current at the battery terminals can be represented by the current drivers $i_{4}(t)=I_{4}$ and $i_{\text {cell }}(t)=I_{\text {app }}$. For simplicity, it is assumed that the battery is cycled with a constant current $I_{\text {app }}$. We also assume that the battery has thin electrodes and, therefore, we can ignore the influence of the battery internal resistance.

[Figure 10 about here.]

The linear graph for the chemical domain is shown in Fig. B.11. We see that the topological graph structure comprises eight edges, to which we have assigned arbitrary sign directions. The number of equations to be solved simultaneously can be reduced by selecting a tree and using a branch-chord formulation, as described in Section 2. Choosing $C_{1}, C_{3}, C_{3}$, $R_{2}$, and $R_{3}$ as branches and $R_{1}, I_{4}$, and $I_{\text {app }}$ as chords can reduce the number of final equations as it will be shown that the equations for $I_{4}$ and $I_{\text {app }}$ are known (i.e., limiting current for oxygen reduction reaction and constant charge/discharge current).

[Figure 11 about here.]

The column matrix of through variables is:

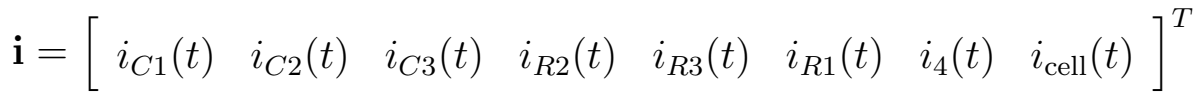

Vector $\mathbf{i}$ can be broken down into the branch through variable vector $\mathbf{i}_{b}$ and chord through variable vector $\mathbf{i}_{c}$ as

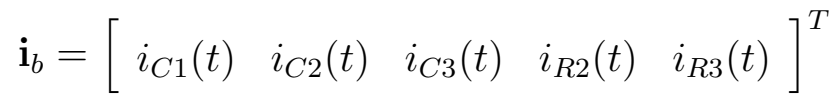




$$
\mathbf{i}_{c}=\left[\begin{array}{lll}
i_{R 1}(t) & i_{4}(t) & i_{\text {cell }}(t)
\end{array}\right]^{T}
$$

Since the currents, which are the derivatives of the electrical charges, are through variables, the charges are also through variables and can be written in vector forms as

$$
\begin{aligned}
& \mathbf{q}_{b}=\left[\begin{array}{lllll}
q_{C 1}(t) & q_{C 2}(t) & q_{C 3}(t) & q_{R 2}(t) & q_{R 3}(t)
\end{array}\right]^{T} \\
& \mathbf{q}_{c}=\left[\begin{array}{lll}
q_{R 1}(t) & q_{4}(t) & q_{\mathrm{cell}}(t)
\end{array}\right]^{T}
\end{aligned}
$$

The current and charge variables are expressed in terms of the current densities and concentrations as follows

$$
\begin{aligned}
& i_{R 1}(t)=A_{\mathrm{pos}} a_{\mathrm{pos}} l_{\mathrm{pos}} j_{1}(t) \\
& i_{R 2}(t)=A_{\mathrm{pos}} a_{\mathrm{pos}} l_{\mathrm{pos}} j_{2}(t) \\
& i_{R 3}(t)=A_{\mathrm{neg}} a_{\mathrm{neg}} l_{\mathrm{neg}} j_{3}(t) \\
& i_{4}(t)=A_{\mathrm{neg}} a_{\mathrm{neg}} l_{\mathrm{neg}} j_{4}(t)
\end{aligned}
$$

and

$$
\begin{array}{ll}
q_{C 1}(t) & =\frac{F A_{\mathrm{pos}} L_{\mathrm{Ni}(\mathrm{OH})_{2}}}{\rho_{\mathrm{Ni}(\mathrm{OH})_{2}}} c_{\mathrm{H}^{+}}(t) \\
q_{C 3}(t) & =\frac{F A_{\text {neg }} L_{\mathrm{MH}}}{\rho_{\mathrm{MH}}} c_{\mathrm{MH}}(t) \\
q_{C 2}(t)+q_{4}(t) & =\frac{F V_{\mathrm{gas}}}{R T(t)} p_{\mathrm{O}_{2}}(t)
\end{array}
$$

Similarly, the across variable vector can be defined as follows

$$
\begin{aligned}
& \mathbf{v}=\left[\begin{array}{llllllll}
v_{C 1}(t) & v_{C 2}(t) & v_{C 3}(t) & v_{R 2}(t) & v_{R 3}(t) & v_{R 1}(t) & v_{4}(t) & v_{\text {cell }}(t)
\end{array}\right]^{T} \\
& \mathbf{v}_{b}=\left[\begin{array}{lllll}
v_{C 1}(t) & v_{C 2}(t) & v_{C 3}(t) & v_{R 2}(t) & v_{R 3}(t)
\end{array}\right]^{T}
\end{aligned}
$$




$$
\mathbf{v}_{c}=\left[\begin{array}{lll}
v_{R 1}(t) & v_{4}(t) & v_{\text {cell }}(t)
\end{array}\right]^{T}
$$

where the voltage variables are related to the battery electrical potentials by

$$
\begin{aligned}
& v_{C k}(t)=\phi_{k}(t) \\
& v_{R k}(t)=\eta_{k}(t) \quad k=1 . .3
\end{aligned}
$$

For convenience, the Butler-Volmer equations are converted into the conductance form by applying the inverse hyperbolic operation to Eqs. (30) as

$$
v_{R k}(t)=\frac{R T(t)}{\alpha_{k} F} \ln \left[\frac{j_{k}(t)}{2 i_{0, k}}+\sqrt{\left(\frac{j_{k}(t)}{2 i_{0, k}}\right)^{2}+1}\right] \quad k=1 . .3
$$

The expressions in Eqs. (27), (29), (28), (34), and (52) can be written as functions of currents, charges, and voltages using the relations in (46), (47), and (51); doing so, we obtain the following set of six terminal equations

$$
\begin{aligned}
v_{C 1}(t)= & U_{1}+\left(T(t)-T_{0}\right) \frac{\partial U_{1}}{\partial T} \\
& +\frac{R T(t)}{F} \ln \left(\frac{c_{\mathrm{H}^{+}, \max } F A_{\mathrm{pos}} L_{\mathrm{Ni}(\mathrm{OH})_{2}}-\rho_{\mathrm{Ni}(\mathrm{OH})_{2} q_{C 1}(t)} c_{e} \rho_{\mathrm{Ni}(\mathrm{OH})_{2} q_{C 1}(t)}}{2 F} \ln \left(\frac{\sqrt{\frac{q_{C 2}(t)+q_{4}(t)}{F V_{\mathrm{gas}}} R T(t)}}{c_{e}^{2}}\right)\right. \\
v_{C 2}(t)= & \left.U_{2}+\left(T(t)-T_{0}\right) \frac{\partial U_{2}}{\partial T}+\frac{R T(t)}{2 F}\right) \\
v_{C 3}(t)= & U_{3}+\left(T(t)-T_{0}\right) \frac{\partial U_{3}}{\partial T}+\frac{R T(t)}{F} \ln \left(c_{e}^{2}\right)+9.712 \times 10^{-4} \\
& +0.2372 \exp \left[-\frac{28.057 \rho_{\mathrm{MH}}}{F A_{\mathrm{neg}} L_{\mathrm{MH}} c_{\mathrm{MH}, \mathrm{max}}} q_{C 3}(t)\right] \\
& -\frac{2.7302 \times 10^{-4}}{\left[\frac{\rho_{\mathrm{MH}}}{F A_{\mathrm{neg}} L_{\mathrm{MH}} c_{\mathrm{MH}, \mathrm{max}}} q_{C 3}(t)\right]^{2}+0.010768} \\
v_{R 2}(t)= & \frac{R T(t)}{\alpha_{2} F} \ln \left[\frac{i_{R 2}(t)}{2 i_{0,2} A_{\mathrm{pos}} a_{\mathrm{pos}} l_{\mathrm{pos}}}+\sqrt{\left(\frac{i_{R 2}(t)}{2 i_{0,2} A_{\mathrm{pos}} a_{\mathrm{pos}} l_{\mathrm{pos}}}\right)^{2}+1}\right. \\
v_{R 3}(t)= & \frac{R T(t)}{\alpha_{3} F} \ln \left[\frac{i_{R 3}(t)}{2 i_{0,3} A_{\mathrm{neg}} a_{\mathrm{neg}} l_{\mathrm{neg}}}+\sqrt{\left(\frac{i_{R 3}(t)}{2 i_{0,3} A_{\mathrm{neg}} a_{\mathrm{neg}} l_{\mathrm{neg}}}\right)^{2}+1}\right] \\
v_{R 1}(t)= & \frac{R T(t)}{\alpha_{1} F} \ln \left[\frac{i_{R 1}(t)}{2 i_{0,1} A_{\mathrm{pos}} a_{\mathrm{pos}} l_{\mathrm{pos}}}+\sqrt{\left(\frac{i_{R 1}(t)}{2 i_{0,1} A_{\mathrm{pos}} a_{\mathrm{pos}} l_{\mathrm{pos}}}\right)^{2}+1}\right]
\end{aligned}
$$


In a similar manner, the current driver in (34) can also be written as

$$
i_{4}(t)=-\frac{q_{C 2}(t)+q_{4}(t)}{p_{\mathrm{O}_{2}, \mathrm{ref}} F V_{\text {gas }}} R T(t) i_{0,4}
$$

We now define the f-cutset and f-circuit matrices. For the graph given in Fig. B.11 and the given tree selection, the fundamental-cutset matrix is obtained:

$$
\mathbf{A}_{f}=\left[\begin{array}{cccccccc}
1 & 0 & 0 & 0 & 0 & -1 & 0 & 0 \\
0 & 1 & 0 & 0 & 0 & 1 & 0 & -1 \\
0 & 0 & 1 & 0 & 0 & 0 & 1 & 1 \\
0 & 0 & 0 & 1 & 0 & 1 & 0 & -1 \\
0 & 0 & 0 & 0 & 1 & 0 & 1 & 1
\end{array}\right]=\left[\begin{array}{cc}
\mathbf{1}_{b} & \mathbf{A}_{c}
\end{array}\right]
$$

which can be used to write the chord transformation equation for this system

$$
\left(\begin{array}{c}
i_{C 1}(t) \\
i_{C 2}(t) \\
i_{C 3}(t) \\
i_{R 2}(t) \\
i_{R 3}(t)
\end{array}\right)=-\left[\begin{array}{ccc}
-1 & 0 & 0 \\
1 & 0 & -1 \\
0 & 1 & 1 \\
1 & 0 & -1 \\
0 & 1 & 1
\end{array}\right]\left(\begin{array}{c}
i_{R 1}(t) \\
i_{4}(t) \\
i_{\text {cell }}(t)
\end{array}\right)
$$

For the charge variables, initial values must be included in the chord transformation equations, which yields

$$
\left(\begin{array}{c}
q_{C 1}(t)-q_{C 1}(0) \\
q_{C 2}(t)-q_{C 2}(0) \\
q_{C 3}(t)-q_{C 3}(0) \\
q_{R 2}(t)-q_{R 2}(0) \\
q_{R 3}(t)-q_{R 3}(0)
\end{array}\right)=-\left[\begin{array}{ccc}
-1 & 0 & 0 \\
1 & 0 & -1 \\
0 & 1 & 1 \\
1 & 0 & -1 \\
0 & 1 & 1
\end{array}\right]\left(\begin{array}{c}
q_{R 1}(t)-q_{R 1}(0) \\
q_{4}(t)-q_{4}(0) \\
q_{\text {cell }}(t)-q_{\text {cell }}(0)
\end{array}\right)
$$


From the fundamental-cutset matrix, one can directly obtain the following fundamental-circuit matrix:

$$
\mathbf{B}_{f}=\left[\begin{array}{cccccccc}
1 & -1 & 0 & -1 & 0 & 1 & 0 & 0 \\
0 & 0 & -1 & 0 & -1 & 0 & 1 & 0 \\
0 & 1 & -1 & 1 & -1 & 0 & 0 & 1
\end{array}\right]=\left[\begin{array}{ll}
\mathbf{B}_{b} & \mathbf{1}_{c}
\end{array}\right]
$$

in which each row corresponds to an equation along the edges enclosing each circuit.

Similarly, making use of Eq. (4), the branch transformation for the system can be written explicitly as

$$
\left(\begin{array}{c}
v_{R 1} \\
v_{4} \\
v_{\text {cell }}
\end{array}\right)=-\left[\begin{array}{ccccc}
1 & -1 & 0 & -1 & 0 \\
0 & 0 & -1 & 0 & -1 \\
0 & 1 & -1 & 1 & -1
\end{array}\right]\left(\begin{array}{c}
v_{C 1} \\
v_{C 2} \\
v_{C 3} \\
v_{R 2} \\
v_{R 3}
\end{array}\right)
$$

Substituting Eq. (47) and the first equation in (59) into the terminal equations in (53) we obtain

$$
\begin{aligned}
& \frac{R T(t)}{\alpha_{1} F} \ln \left[\frac{i_{R 1}(t)}{2 i_{0,1} A_{\mathrm{pos}} a_{\mathrm{pos}} l_{\mathrm{pos}}}+\sqrt{\left(\frac{i_{R 1}(t)}{2 i_{0,1} A_{\mathrm{pos}} a_{\mathrm{pos}} l_{\mathrm{pos}}}\right)^{2}+1}\right]+
\end{aligned}
$$

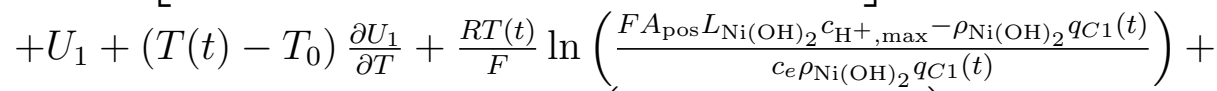

$$
\begin{aligned}
& -U_{2}+\left(T(t)-T_{0}\right) \frac{\partial U_{2}}{\partial T}+\frac{R T(t)}{2 F} \ln \left(\frac{\sqrt{R T(t)\left(q_{C 2}(t)+q_{4}(t)\right)}}{c_{e}^{2} \sqrt{F V_{\mathrm{gas}}}}\right)+ \\
& -\frac{R T(t)}{\alpha_{2} F} \ln \left[\frac{i_{R 2}(t)}{2 i_{0,2} A_{\mathrm{pos}} a_{\mathrm{pos}} l_{\mathrm{pos}}}+\sqrt{\left(\frac{i_{R 2}(t)}{2 i_{0,2} A_{\mathrm{pos}} a_{\mathrm{pos}} l_{\mathrm{pos}}}\right)^{2}+1}\right] \quad=0
\end{aligned}
$$


By applying the chord transformations in (56) and (57) to both current and charge variables in (54) and (60), we obtain the following set of two equations:

$$
\left\{\begin{array}{l}
-\frac{A_{\mathrm{neg}} a_{\mathrm{neg}} l_{\mathrm{neg}}\left[q_{\mathrm{cell}}(t)+q_{C 2}(0)-q_{R 1}(t)+q_{R 1}(0)-q_{\mathrm{cell}}(0)+q_{4}(t)\right] R T(t) i_{0,4}}{F V_{\mathrm{gas}} p_{\mathrm{O}_{2}, \mathrm{ref}}}=i_{4}(t) \\
\frac{R T(t)}{\alpha_{1} F} \ln \left[\frac{i_{R 1}(t)}{2 i_{0,1} A_{\mathrm{pos}} a_{\mathrm{pos}} l_{\mathrm{pos}}}+\sqrt{\left(\frac{i_{R 1}(t)}{2 i_{0,1} A_{\mathrm{pos}} a_{\mathrm{pos}} l_{\mathrm{pos}}}\right)^{2}+1}\right]+U_{1}+\left(T(t)-T_{0}\right) \frac{\partial U_{1}}{\partial T}+ \\
+\frac{R T(t)}{F} \ln \left(\frac{\left.F A_{\mathrm{pos}} L_{\mathrm{Ni}(\mathrm{OH})_{2}} c_{\mathrm{H}}+,{ }_{\max }-\rho_{\mathrm{Ni}(\mathrm{OH})_{2}\left(q_{R 1}(t)+q_{R 1}(0)-q_{C 1}(0)\right)}\right)+}{c_{e} \rho_{\mathrm{Ni}(\mathrm{OH})_{2}}\left(q_{R 1}(t)+q_{R 1}(0)-q_{C 1}(0)\right)}\right)+ \\
-U_{2}+\left(T(t)-T_{0}\right) \frac{\partial U_{2}}{\partial T}+\frac{R T(t)}{2 F} \ln \left(\frac{\sqrt{R T(t)\left(-q_{R 1}(t)+q_{R 1}(0)+q_{\mathrm{cell}}(t)-q_{\mathrm{cell}}(0)+q_{C 2}(0)+q_{4}(t)\right)}}{c_{e}^{2} \sqrt{F V_{\mathrm{gas}}}}\right)+ \\
-\frac{R T(t)}{\alpha_{2} F} \ln \left[\frac{-i_{R 1}(t)+i_{\mathrm{cell}}(t)}{2 i_{0,2} A_{\mathrm{pos}} a_{\mathrm{pos}} l_{\mathrm{pos}}}+\sqrt{\left(\frac{-i_{R 1}(t)+i_{\mathrm{cell}}(t)}{2 i_{0,2} A_{\mathrm{pos}} a_{\mathrm{pos}} l_{\mathrm{pos}}}\right)^{2}+1}\right]=0
\end{array}\right.
$$

It should be noted from the above equation that $i_{\text {cell }}(t)$ is the current applied on the battery terminals and, therefore, is completely known. We have the following relationships

$$
\begin{aligned}
& i_{R 1}(t)=\frac{d q_{R 1}(t)}{d t} \\
& i_{4}(t)=\frac{d q_{4}(t)}{d t} \\
& i_{\text {cell }}(t)=\frac{d q_{\text {cell }}(t)}{d t}
\end{aligned}
$$

Therefore, Eq. (61) become a set of two ordinary differential equations (ODEs) which only consists of three unknowns $q_{R 1}(t), q_{4}(t)$, and $T(t)$ and can easily be solved using a numerical integrator with proper initial conditions, if the temperature is known.

\subsection{Linear graph for thermal domain}

In Section 4.2 we have shown the steps to develop a linear graph and system equations for a NiMH model under the assumption of constant battery 
temperature. In real automotive applications, studying the thermal effects of batteries is of particular importance due to the large influence of battery temperature on the battery and vehicle performance. Besides reducing battery efficiency, overheating a battery may even cause an explosion if the battery temperature is not controlled. Due to these reasons, it is desirable to also develop a linear graph for the thermal effects in a car battery.

To construct a linear graph for the thermal domain, we can make use of the entropy-temperature relationship in Eq. (16) for the four chemical reactions. Considering the time-derivative of the entropies as through variables and the temperature as an across variable, we can construct the linear graph for the thermal domain as shown in Fig. B.12. By applying the branch and chord transformations in Eqs. (59), (56), and (57), we can also convert Eq. (16) into a function that is only dependent on $T(t), q_{R 1}(t)$, and $q_{4}(t)$.

[Figure 12 about here.]

\subsection{Simulation results}

The model developed has been used to simulate several scenarios in order to observe its behaviors, particularly with regard to thermal effects. Table B.2 contains the model parameters used in the simulations. These parameters were identified using homotopy optimization from a $3.4 A h \mathrm{NiMH}$ battery produced by North American Battery Company (NABC) based on the reference parameters provided in the work of $\mathrm{Wu}$ et al. (2001). The battery data was measured at A\&D Technology's laboratory in Ann Arbor, Michigan, USA.

The battery voltage versus time for four different discharge and charge rates from $1 C(3.4 A)$ to $1 / 8 C(0.425 A)$ are shown in Fig. B.13 and 
Fig. B.14. As expected, the battery voltage drops/rises more quickly as the discharge/charge current is increased. Figure B.15 compares the battery voltages obtained from simulation results and battery testing data at a constant charge and discharge rate of $1 / 5 C$. It can be seen that these results are in good agreement.

[Figure 13 about here.]

[Figure 14 about here.]

[Figure 15 about here.]

The battery temperature during discharge and charge versus time is shown in Fig. B.16. It is assumed that the battery has been cooled down to

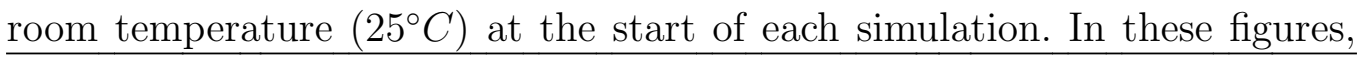
the depletion of reactants results in high over-potential loss that causes a rapid cell temperature increase. Due to the temperature exchange with the environment, the battery temperature is flattened out at the end of the cycle. At a high charge rate, due to the high ohmic and over-potential losses, $\underline{\text { high charge potential is needed as expected. The cell heat generation is also }}$ significant at high discharge currents for the same reasons. This may cause the cell temperature to rise more than $10^{\circ} \mathrm{C}$. At high-rate charge, the oxygen generation also increases very quickly, contributing significantly to the increase in the cell temperature. However, during discharge, oxygen gas is only generated at low currents. This explains the difference between the cell temperatures in the two figures at the same rates, particularly at the high rates. $\underline{\text { Battery temperature control is therefore very important, particularly in a }}$ 
battery electric vehicle or hybrid electric vehicle system where the current intensity is usually very high.

One of the most noticeable results is that the linear graph model simulates approximately $30 \%$ faster than the lumped model (i.e., all equations in $\mathrm{Wu}$ et al. (2001) are stacked together) at all charge and discharge rates as summarized in Table B.3. The simulation times were obtained from a Dell $^{\mathrm{TM}}$ OptiPlex $2.9 \mathrm{GHz}$ desktop computer using Maple dsolve function based on the default Runge-Kutta Fehlberg numeric integrator with the same settings $\left(\right.$ abserr $=$ relerr $\left.=10^{-7}\right)$ for both linear graph and lumped models.

[Figure 16 about here.]

[Figure 17 about here.]

[Table 1 about here.]

\section{Conclusion}

In this paper, we have presented a linear graph formulation for systematically generating a compact set of dynamic equations governing electrochemical systems. By carefully managing how equations are formed, a smaller set of expressions is obtained. This benefits symbolic implementation by reducing the size of the largest expression that needs to be handled by the computer, thus allowing for the analysis of more complicated systems. It was also shown that the equations obtained using linear graph theoretical approach simulated approximately $30 \%$ faster than the original lumped model.

Since the interconnections between a system's components are represented by a linear graph, tree selection strategies can be used to determine 
the modeling variables for the system. It is clear that this flexibility can provide benefits during formulation as well as simulation.

This approach can be extended to modeling a more complex system such as a battery electric vehicle or a hybrid electric vehicle within which a battery model is an important part. This is a potential for future research since modeling individual parts of a battery/hybrid electric vehicle has been done in the literature (McPhee et al. , 2004; Schmitke et al. , 2008; Dao et al. , 2010), but never before using linear graph theory.

\section{Acknowledgment}

Financial support for this research has been provided by the Natural Sciences and Engineering Research Council of Canada (NSERC), Toyota, and Maplesoft.

\section{References}

Paxton, B. and Newman, J., "Modeling of nickel/metal hydride batteries," J. Electrochem. Soc., Vol. 144, No. 11, pp.3818-3831, 1997.

Newman, J. and William, T., Porous-electrode theory with battery applications, AIChE Journal, Vol. 21, No. 1, pp.25-41, 1975.

Newman, J. and Thomas-Alyea, K.E., Electrochemical Systems, 3rd ed, John Wiley \& Sons Inc., 2004.

Wu, B., Mohammed, M., Brigham, D., Elder, R., and White, R.E., A nonisothermal model of a nickel-metal hydride cell, Journal of Power Sources, Vol. 101, No. 2, pp.149-157, 2001. 
Salameh, Z.M., Casacca, M.A., and Lynch, W.A., A mathematical model for lead-acid batteries, IEEE Trans. On Energy Conversion, Vol. 7, No. 1, pp.93-98, 1992.

Chen, M. and Rinćon-Mora, G.A., "Accurate electrical battery model capable of predicting runtime and I-V performance," IEEE Trans. On Energy Conversion, Vol. 21, No. 2, pp.504-511, 2006.

Roe, P.H., Networks and Systems, Addison-Vesley, 1966.

Andrews, G. and Kesavan, H., The vector-network model: a new approach to vector dynamics, Mech. Mach. Theory, Vol. 10, No. 1, pp.57-75, 1975.

McPhee, J., Schmitke, C., and Redmond, S., Dynamic modelling of mechatronic multibody systems with symbolic computing and linear graph theory, Mathematical and Computer Modelling of Dynamical Systems, Vol. 10, No. 1, pp.1-23, 2004.

McPhee, J., On the use of linear graph theory in multibody system dynamics, Nonlinear Dynamics, Vol. 9, pp.73-90, 1996.

McPhee, J., Automatic generation of motion equations for planar mechanical systems using the new set of 'branch' coordinates, Mech. Mach. Theory, Vol. 33, No. 6, pp.805-823, 1998.

Schmitke, C., Morency, K., and McPhee, J., Using graph theory and symbolic computing to genete efficient models for multi-body vehicle dynamics, $J$. of Multi-body Dynamics, Part K, Vol. 222, No. 4, pp.339-352, 2008. 
Léger, M., and McPhee, J., Selection of modeling coordinates for forward dynamic multibody simulations, Multibody Syst. Dyn., Vol. 18, No. 2, pp.277$297,2007$.

Wang, Z.H., Gu, W.B., and Wang C.Y., Simulation of thermal runaway in lithium-ion batteries, in Proceedings of the 196th ECS Fall Meeting, Honolulu, Hawaii, 1999.

Gomadam, P.M., Weidner, J.W., and White, R.E., Modeling heat conduction in spiral geometries, Journal of Electrochemical Society, Vol. 150, No. 10, pp.A1339-A1345, 2003.

Gu, W. B., Wang, C. Y., Li, S., Geng, M. M., and Liaw, B. Y., Modeling discharge and charge characteristics of nickel-metal hydride batteries, Electrochim. Acta, Vol. 44, pp.4525-4541, 1999.

Albertus, P., Christensen, J., and Newman, J., Modeling side reactions and nonisothermal effects in nickel metal-hydride batteries, Journal of Electrochemical Society, Vol. 155, No. 1, pp.A48-A60, 2008.

Dao, T.-S., Seaman, A., and McPhee, J., Mathematics-based modeling of a series-hybrid electric vehicle, Proc. of the 5th Asian Conference on Multibody Dynamics, 2010. 
Appendix A. Through and across variables for some physical systems

\begin{tabular}{|c|c|c|}
\hline Domain & Through [unit] & Across [unit] \\
\hline \multirow{2}{*}{ Electrical } & current & voltage \\
\hline & $i[A]$ & $v[V]$ \\
\hline \multirow[t]{2}{*}{ Mechanical translational } & force & velocity \\
\hline & $\vec{F}[N]$ & $\vec{v}\left[m . s^{-1}\right]$ \\
\hline \multirow[t]{2}{*}{ Mechanical rotational } & torque & angular velocity \\
\hline & $\vec{\tau}[N . m]$ & $\vec{\omega}\left[\mathrm{rad} . \mathrm{s}^{-1}\right]$ \\
\hline \multirow[t]{2}{*}{ Hydraulic } & volume flow & pressure \\
\hline & $\phi_{v}\left[m^{3} \cdot s^{-1}\right]$ & $p\left[N . m^{-2}\right]$ \\
\hline \multirow[t]{2}{*}{ Thermal } & entropy flow & temperature \\
\hline & $\frac{d S}{d t}\left[W .^{\circ} K^{-1}\right]$ & $T\left[{ }^{\circ} K\right]$ \\
\hline \multirow[t]{2}{*}{ Chemical } & molar flow & chemical potential \\
\hline & $\frac{d N}{d t}\left[\right.$ mol.s $\left.s^{-1}\right]$ & $\mu\left[J \mathrm{~mol}^{-1}\right]$ \\
\hline
\end{tabular}

Table A.1: Through and across variables. 


\section{Appendix B. NiMH battery parameters used in simulations}

\begin{tabular}{|c|c|c|c|}
\hline Parameter & Unit & Symbol & Value \\
\hline Specific electrode area of positive electrode & $\mathrm{cm}^{2} \cdot \mathrm{cm}^{-3}$ & $a_{\text {pos }}$ & 4000.0 \\
\hline $\begin{array}{l}\text { Specific electrode area of negative elec- } \\
\text { trode }\end{array}$ & $\mathrm{cm}^{2} . \mathrm{cm}^{-3}$ & $a_{\text {neg }}$ & 3000.0 \\
\hline Surface area of positive electrode & $\mathrm{cm}^{2}$ & $A_{\text {pos }}$ & 175.0 \\
\hline Surface area of negative electrode & $\mathrm{cm}^{2}$ & $A_{\text {neg }}$ & 100.0 \\
\hline Thickness of positive electrode & $\mathrm{cm}$ & $l_{\mathrm{pos}}$ & $3.3 \times 10^{-2}$ \\
\hline Thickness of negative electrode & $\mathrm{cm}$ & $l_{\text {neg }}$ & $2.8 \times 10^{-2}$ \\
\hline Loading of nickel active material & g.cm ${ }^{-2}$ & $L_{\mathrm{Ni}(\mathrm{OH})_{2}}$ & $6.8 \times 10^{-2}$ \\
\hline Loading of metal hydride material & g.cm ${ }^{-2}$ & $L_{\mathrm{MH}}$ & $1.13 \times 10^{-1}$ \\
\hline Concentration of $\mathrm{KOH}$ electrolyte & mol.cm ${ }^{-3}$ & $c_{e}$ & $7.0 \times 10^{-3}$ \\
\hline $\begin{array}{l}\text { Reference concentration of } \mathrm{KOH} \text { elec- } \\
\text { trolyte }\end{array}$ & mol.cm ${ }^{-3}$ & $c_{e, \mathrm{ref}}$ & $1.0 \times 10^{-3}$ \\
\hline $\begin{array}{l}\text { Maximum concentration of } \mathrm{Ni}(\mathrm{OH})_{2} \\
\text { in nickel active material }\end{array}$ & mol.cm ${ }^{-3}$ & $c_{\mathrm{H}^{+}, \max }$ & $3.7 \times 10^{-2}$ \\
\hline $\begin{array}{l}\text { Reference concentration of } \mathrm{Ni}(\mathrm{OH})_{2} \\
\text { in nickel active material }\end{array}$ & mol.cm ${ }^{-3}$ & $c_{\mathrm{H}^{+}, \mathrm{ref}}$ & $0.5 c_{\mathrm{H}^{+}, \max }$ \\
\hline $\begin{array}{l}\text { Maximum concentration of hydrogen } \\
\text { in metal hydride material }\end{array}$ & mol.cm ${ }^{-3}$ & $c_{\mathrm{MH}, \max }$ & $1.0 \times 10^{-1}$ \\
\hline
\end{tabular}




\begin{tabular}{|c|c|c|c|}
\hline $\begin{array}{l}\text { Reference concentration of hydrogen } \\
\text { in metal hydride material }\end{array}$ & mol.cm ${ }^{-3}$ & $c_{\mathrm{MH}, \mathrm{ref}}$ & $0.5 c_{\mathrm{MH}, \max }$ \\
\hline Reference oxygen pressure & atm & $p_{\mathrm{O}_{2}, \text { ref }}$ & 1.0 \\
\hline \multicolumn{4}{|l|}{$\begin{array}{l}\text { Exchange current density of reaction at } \\
\text { reference }\end{array}$} \\
\hline reactant concentration for first reaction & A.cm ${ }^{-2}$ & $i_{0,1, \text { ref }}$ & $15.1 \times 10^{-4}$ \\
\hline \multicolumn{4}{|l|}{$\begin{array}{l}\text { Exchange current density of reaction at } \\
\text { reference }\end{array}$} \\
\hline reactant concentration for second reaction & A.cm ${ }^{-2}$ & $i_{0,2, \text { ref }}$ & $2.0 \times 10^{-4}$ \\
\hline \multicolumn{4}{|l|}{$\begin{array}{l}\text { Exchange current density of reaction at } \\
\text { reference }\end{array}$} \\
\hline reactant concentration for third reaction & A.cm ${ }^{-2}$ & $i_{0,3, \text { ref }}$ & $10.2 \times 10^{-4}$ \\
\hline \multicolumn{4}{|l|}{$\begin{array}{l}\text { Exchange current density of reaction at } \\
\text { reference }\end{array}$} \\
\hline reactant concentration for fourth reaction & A.cm ${ }^{-2}$ & $i_{0,4, \text { ref }}$ & $13.2 \times 10^{-4}$ \\
\hline Activation energy for first reaction & J.mol ${ }^{-1}$ & $E_{a, 1}$ & $10.0 \times 10^{3}$ \\
\hline Activation energy for second reaction & J.mol ${ }^{-1}$ & $E_{a, 2}$ & $120.0 \times 10^{3}$ \\
\hline Activation energy for third reaction & J.mol ${ }^{-1}$ & $E_{a, 3}$ & $10.0 \times 10^{3}$ \\
\hline Activation energy for fourth reaction & J.mol ${ }^{-1}$ & $E_{a, 4}$ & $10.0 \times 10^{3}$ \\
\hline Reversible heat for first reaction & $V . K^{-1}$ & $\frac{\partial U_{1}}{\partial T}$ & $\begin{array}{l}-1.35 \times \\
10^{-3}\end{array}$ \\
\hline
\end{tabular}




\begin{tabular}{|c|c|c|c|c|}
\hline \multirow[t]{2}{*}{ Reversible heat for second reaction } & \multirow[t]{2}{*}{$V . K^{-1}$} & \multirow[t]{2}{*}{$\frac{\partial U_{2}}{\partial T}$} & -1.68 & \multirow[t]{2}{*}{$x$} \\
\hline & & & $10^{-3}$ & \\
\hline \multirow[t]{2}{*}{ Reversible heat for third reaction } & \multirow[t]{2}{*}{$V \cdot K^{-1}$} & \multirow[t]{2}{*}{$\frac{\partial U_{3}}{\partial T}$} & -1.55 & $x$ \\
\hline & & & $10^{-3}$ & \\
\hline \multirow[t]{2}{*}{ Reversible heat for fourth reaction } & \multirow[t]{2}{*}{$V \cdot K^{-1}$} & \multirow{2}{*}{$\frac{\partial U_{4}}{\partial T}$} & -1.68 & $x$ \\
\hline & & & $10^{-3}$ & \\
\hline Charge transfer coefficient & & $\alpha_{1}$ & 0.5 & \\
\hline Charge transfer coefficient & & $\alpha_{2}$ & 1.0 & \\
\hline Charge transfer coefficient & & $\alpha_{3}$ & 0.5 & \\
\hline \multirow[t]{2}{*}{ Open-circuit voltage } & \multirow[t]{2}{*}{$V$} & \multirow{2}{*}{$U_{1, c}, U_{1, d}$} & 0.527 & \\
\hline & & & 0.458 & \\
\hline Open-circuit voltage & $V$ & $U_{2}$ & 0.4011 & \\
\hline Open-circuit voltage & $V$ & $U_{3}$ & -0.8279 & \\
\hline Open-circuit voltage & $V$ & $U_{4}$ & 0.4011 & \\
\hline Gas volume in NiMH cell & $\mathrm{cm}^{3}$ & $V_{\text {gas }}$ & $1.0 \times 10^{-1}$ & \\
\hline Density of nickel active material & g.cm ${ }^{-3}$ & $\rho_{\mathrm{Ni}(\mathrm{OH})_{2}}$ & 3.4 & \\
\hline Density of metal hydride & g.cm $\mathrm{cm}^{-3}$ & $\rho_{\mathrm{MH}}$ & 7.47 & \\
\hline Reference battery temperature & $K$ & $T_{0}$ & 303.15 & \\
\hline
\end{tabular}

Table B.2: NiMH battery parameters. 
List of Tables 
Table B.3: Average simulation time (in seconds) comparison between linear graph model and lumped model.

\begin{tabular}{lcc}
\hline Applied current & Linear graph model & Lumped model \\
\hline Discharge 1 $C$ & 0.898 & 1.115 \\
Discharge 1/2 $C$ & 0.922 & 1.176 \\
Discharge 1/4 $C$ & 0.902 & 1.121 \\
Discharge 1/8 $C$ & 0.916 & 1.198 \\
Charge 1 $C$ & 0.904 & 1.128 \\
Charge 1/2 $C$ & 0.919 & 1.122 \\
Charge 1/4 $C$ & 0.918 & 1.124 \\
Charge 1/8 $C$ & 0.920 & 1.189 \\
\hline
\end{tabular}




\section{List of Figures}




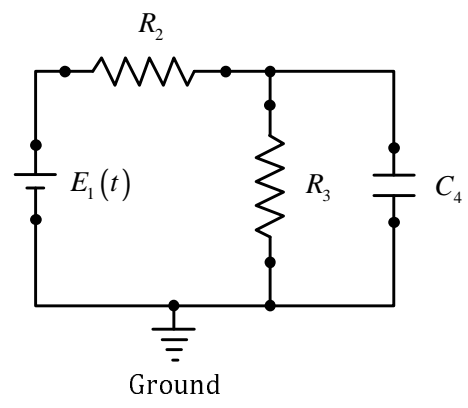

Figure B.1: Electrical network example. 


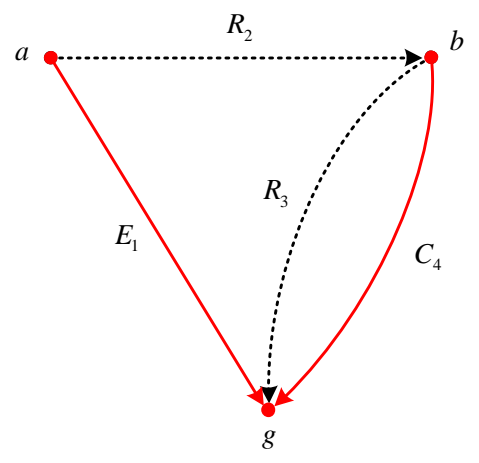

Figure B.2: Linear graph isomorphic to electrical network. 


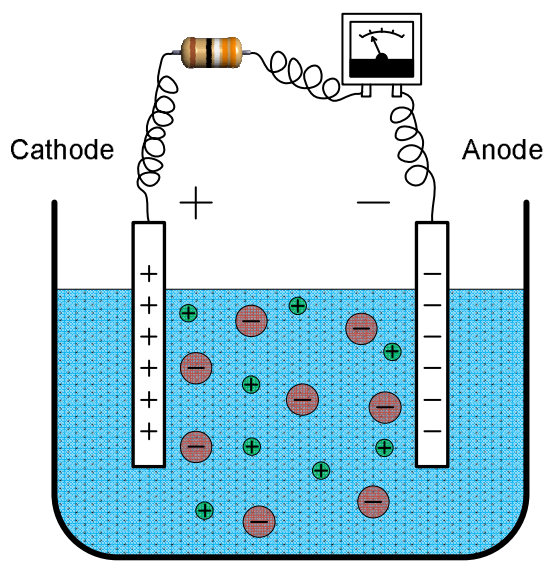

Figure B.3: Electrochemical cell. 


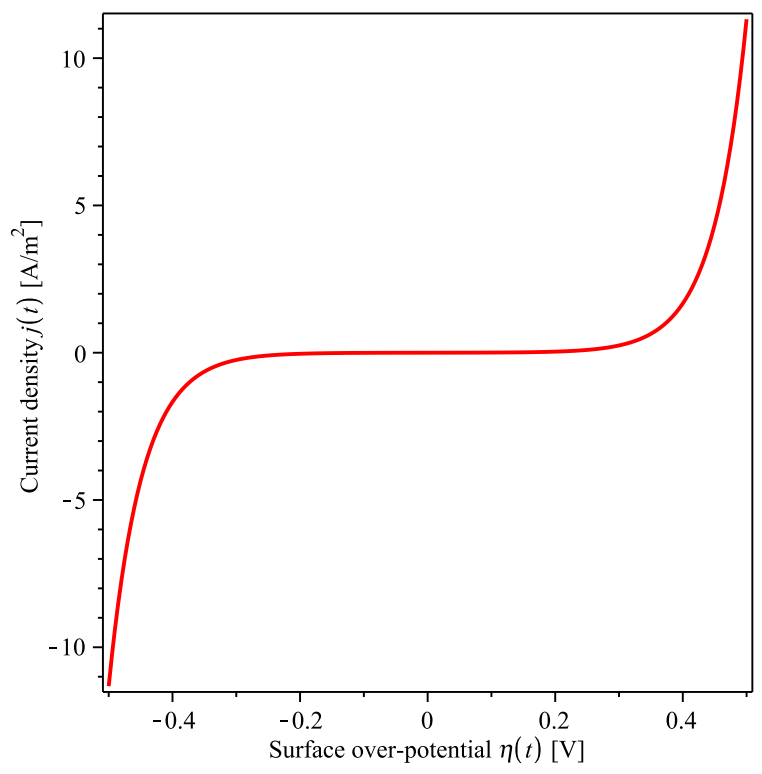

Figure B.4: Dependence of current density on surface over-potential on positive electrode of a $\mathrm{NiMH}$ cell at $30^{\circ} \mathrm{C}$. 


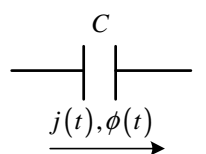

(a)

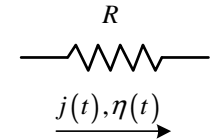

(b)

Figure B.5: Circuitry representation for (a) open-circuit potential equation and (b) ButlerVolmer's equation. 


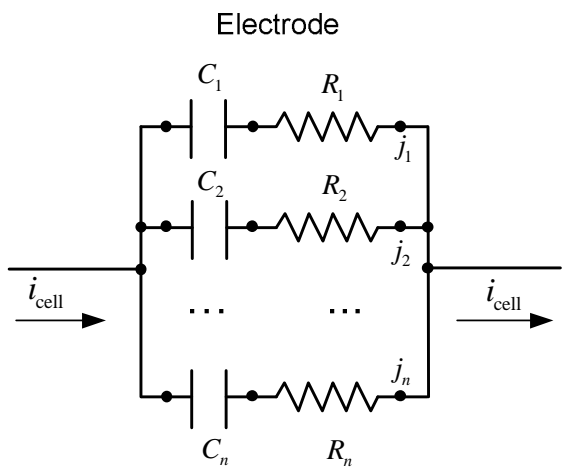

Figure B.6: Circuitry representation for multiple reactions. 


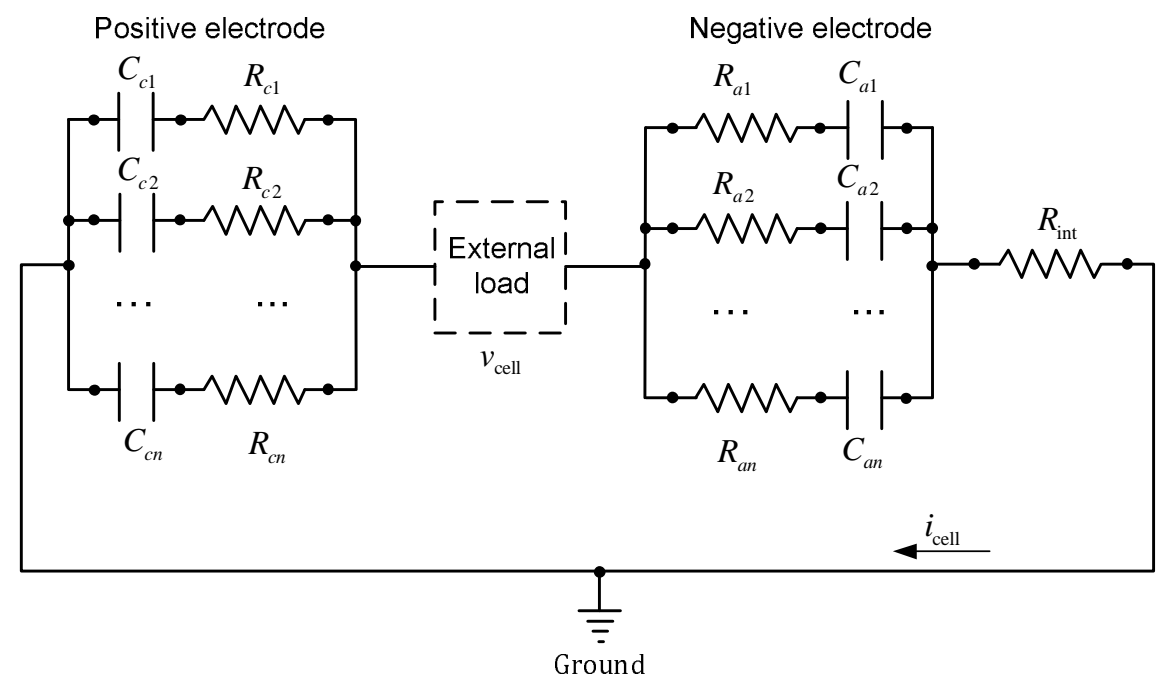

Figure B.7: Equivalent circuit-based representation for electrochemical cell. 


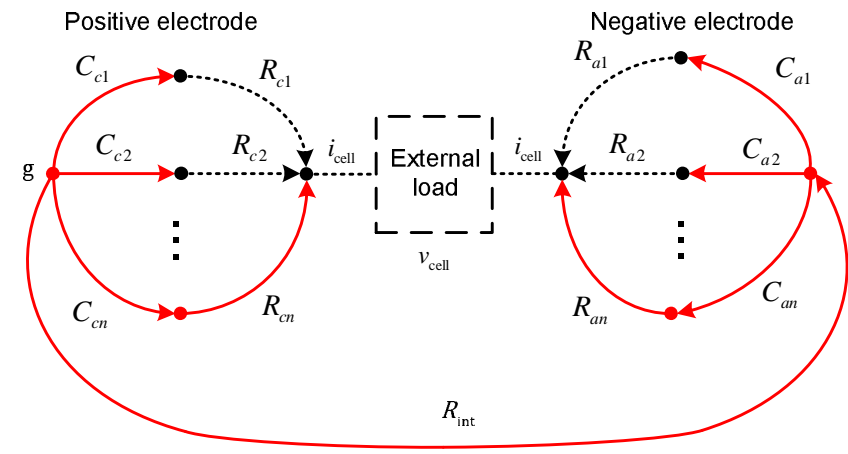

(a)

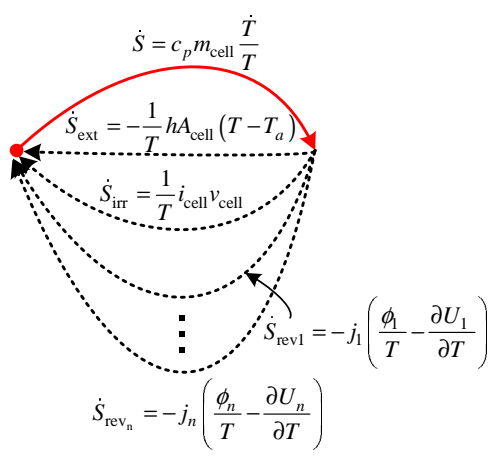

(b)

Figure B.8: Linear graph representation for (a) chemical domain and (b) thermal domain. 


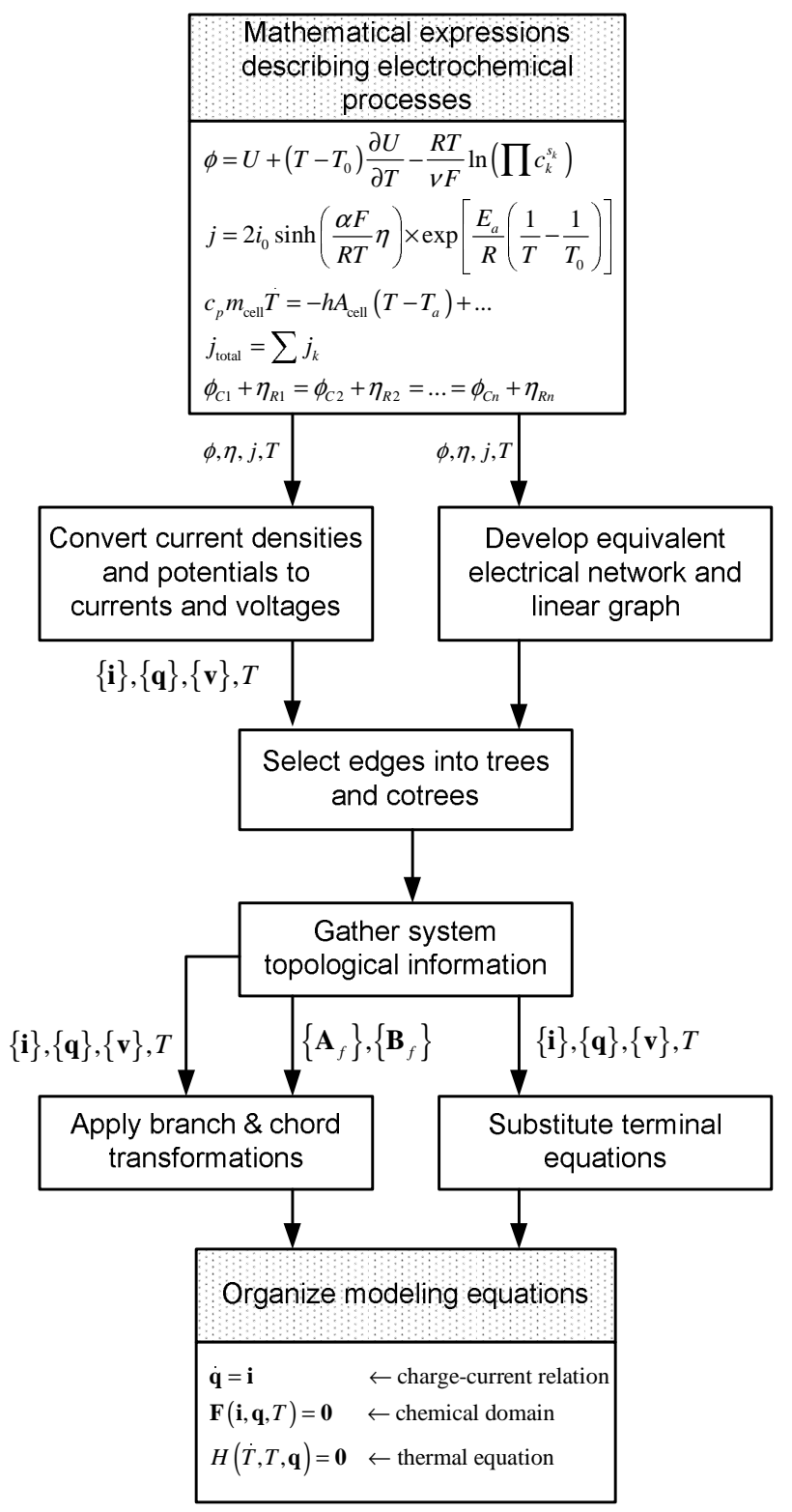

Figure B.9: Formulation steps for electrochemical cells. 


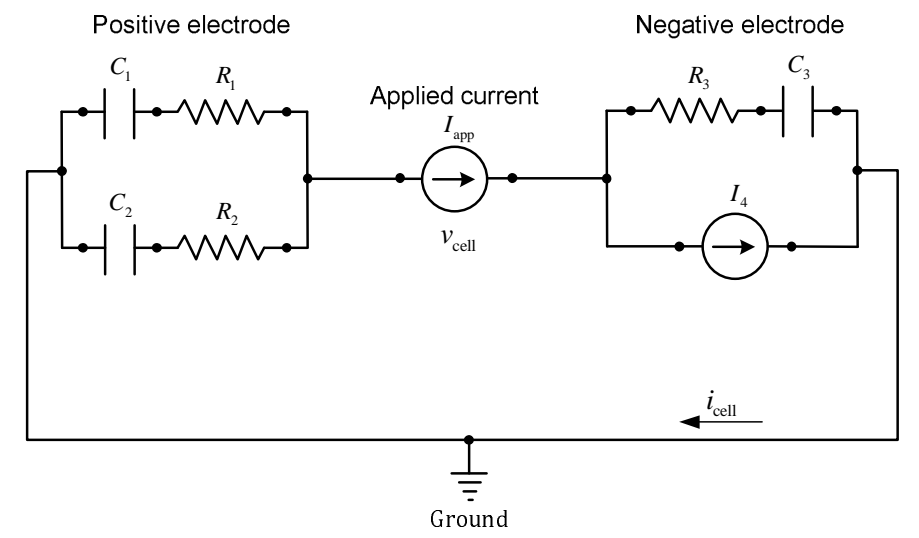

Figure B.10: Circuitry representation for NiMH cell. 


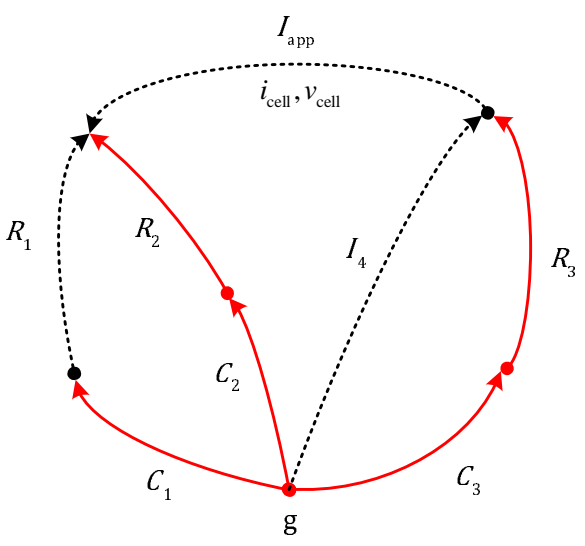

Figure B.11: Linear graph presentation for main and side chemical reactions. 


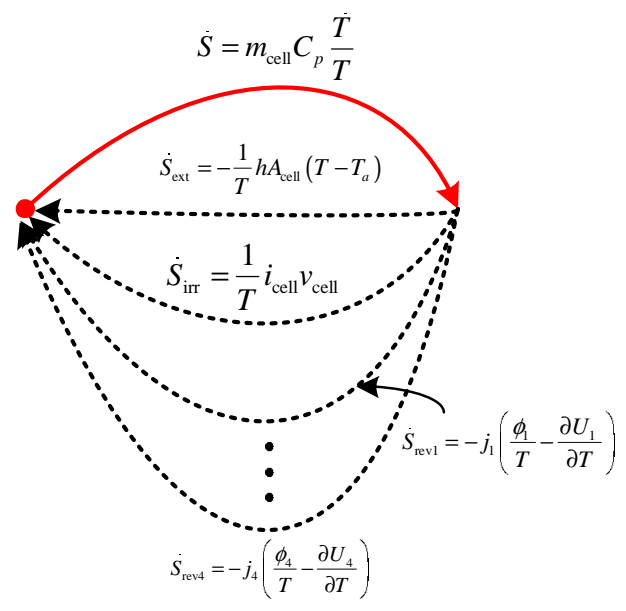

Figure B.12: Linear graph representation for thermal domain. 


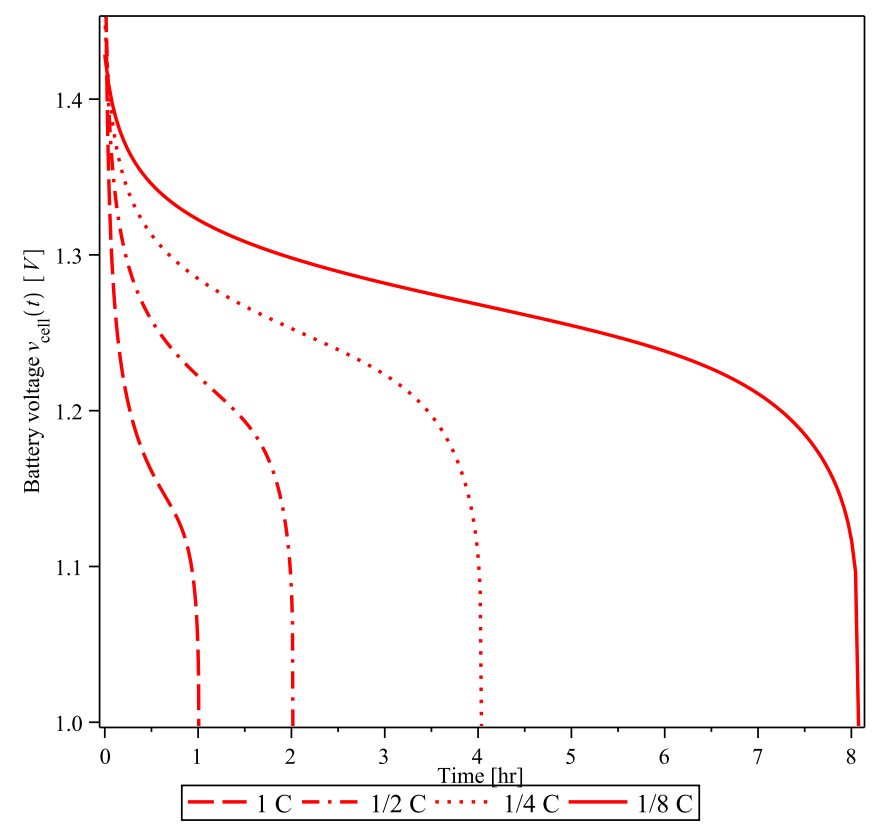

Figure B.13: Battery discharge at constant rates. 


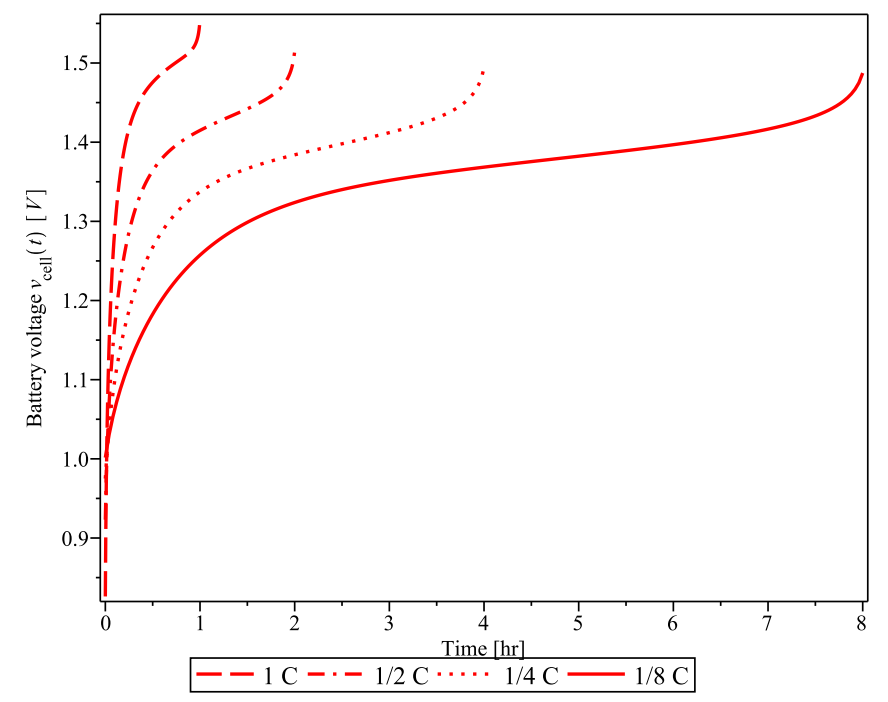

Figure B.14: Battery charge at constant rates. 


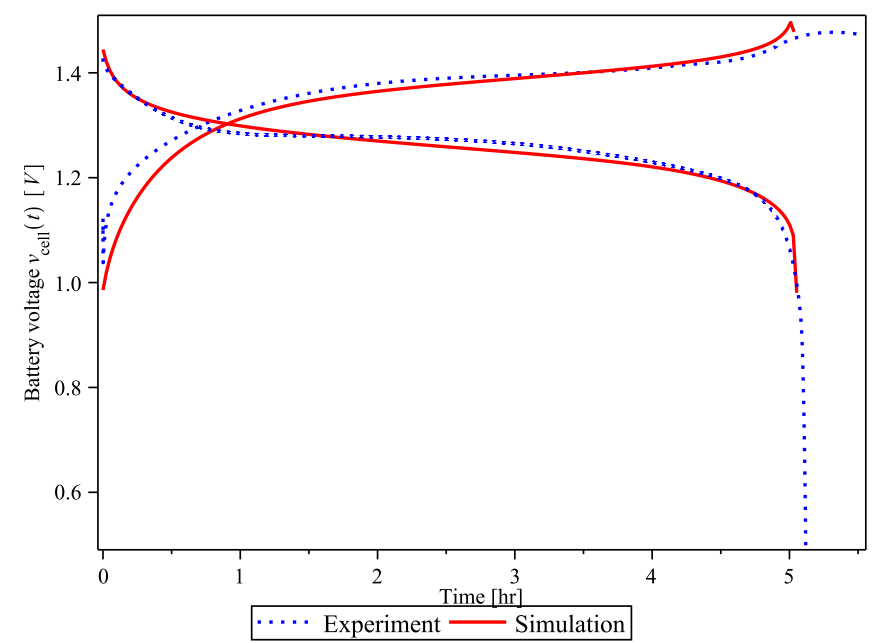

Figure B.15: Simulated and experimental battery voltages at $1 / 5 \mathrm{C}$ charge/discharge rate. 


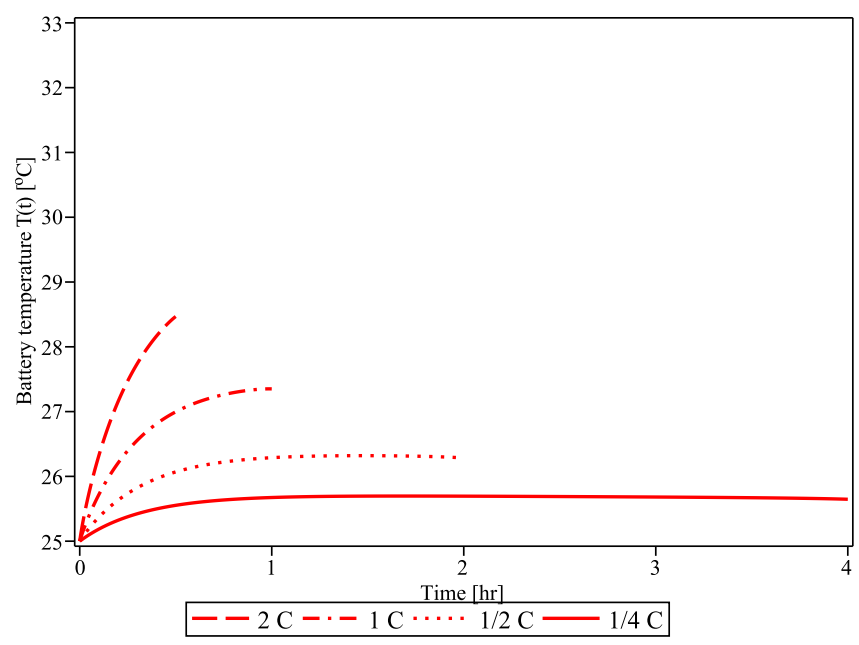

Figure B.16: Battery temperature rising from initial temperature $25^{\circ} \mathrm{C}$ at constant discharge rates. 


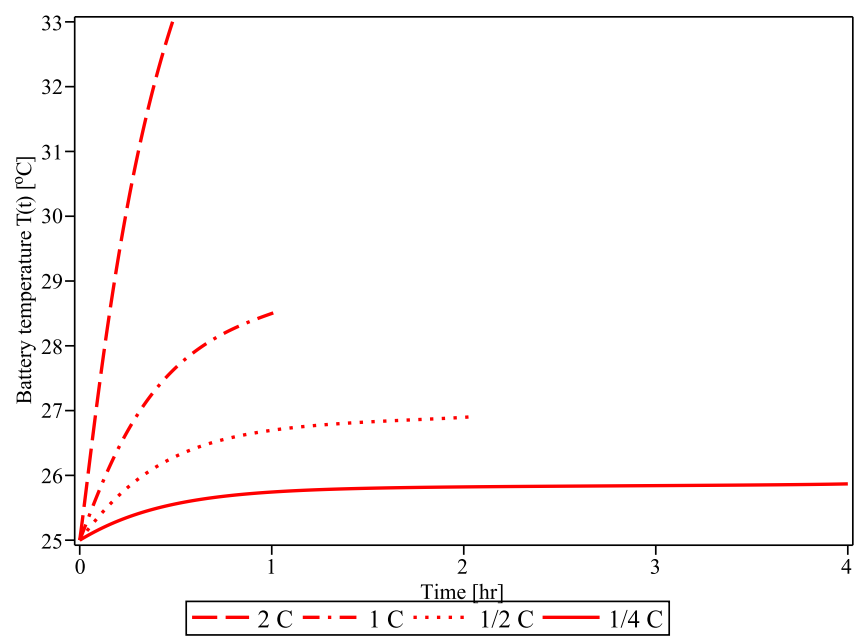

Figure B.17: Battery temperature rising from initial temperature $25^{\circ} \mathrm{C}$ at constant charge rates. 